\title{
A HAMILTONIAN PRESERVING DISCONTINUOUS GALERKIN METHOD FOR THE GENERALIZED KORTEWEG-DE VRIES EQUATION
}

\author{
HAILIANG LIU ${ }^{\dagger}$ AND NIANYU YI ${ }^{\ddagger}$
}

\begin{abstract}
The invariant preserving property is one of the guiding principles for numerical algorithms in solving wave equations, in order to minimize phase and amplitude errors after long time simulation. In this paper, we design, analyze and numerically validate a Hamiltonian preserving discontinuous Galerkin method for solving the Korteweg-de Vries (KdV) equation. For the generalized $\mathrm{KdV}$ equation, the semi-discrete formulation is shown to preserve both the first and the third conserved integrals, and approximately preserve the second conserved integral; for the linearized $\mathrm{KdV}$ equation, all the first three conserved integrals are preserved, and optimal error estimates are obtained for polynomials of even degree. The preservation properties are also maintained by the fully discrete DG scheme. Our numerical experiments demonstrate both high accuracy of convergence and preservation of all three conserved integrals for the generalized KdV equation. We also show that the shape of the solution, after long time simulation, is well preserved due to the Hamiltonian preserving property.
\end{abstract}

\section{INTRODUCTION}

This paper is the continuation of our project, initiated in [40], of developing a high order discontinuous Galerkin (DG) method to preserve some key invariants for the generalized Korteweg-de Vries (gKdV) [18] equation:

$$
u_{t}+f(u)_{x}+\epsilon u_{x x x}=0, \quad x \in \mathbb{R}, t>0,
$$

where the subscript $t$ (or $x$, respectively) denotes the differentiation with respect to time variable $t$ (or $x$ ), $\epsilon$ is a given constant, and $f$ is a smooth function. This is a nonlinear, dispersive partial differential equation for $u$ of two real variables. The original form of the KdV equation corresponds to (1) with $\epsilon=1$ and $f=3 u^{2}$.

The KdV equation has several connections to physical problems. In addition to being the governing equation of the string in the Fermi-Pasta-Ulam problem in the continuum limit, it approximately describes the evolution of long, one-dimensional waves in many physical settings, including shallow-water waves with weakly non-linear restoring forces, long internal waves in a density-stratified ocean, ion-acoustic waves in a plasma, acoustic waves on a crystal lattice, and more.

Dispersion and non-linearity can interact to produce permanent and localized wave forms; It can be shown that any sufficiently fast decaying smooth solution will eventually split into a finite superposition of solitons traveling to the right plus a decaying dispersive part traveling to the left. This was first observed numerically by Zabusky \& Kruskal (1965) [41], long after John Scott Russell's experimental observation of solitons in 1834. One appealing feature of the KdV equation is that it has infinitely many integrals of motion (Miura, Gardner \& Kruskal 1968) [27], which do not change with time. This is also accounted by the fact that the KdV equation can be reformulated into the Lax system, called Lax pairs [20].

2000 Mathematics Subject Classification. 65M60, 65M12, 35Q53.

Key words and phrases. Discontinuous Galerkin method, Korteweg-de Vries equation, conservation, stability. 
The KdV equation is rich in conserved integrals; the first three for (1) are classical:

$$
E_{1}=\int_{\mathbb{R}} u d x, \quad E_{2}=\int_{\mathbb{R}} u^{2} d x, \text { and } E_{3}=\int_{\mathbb{R}}\left(\frac{\epsilon}{2} u_{x}^{2}-\int^{u} f(\xi) d \xi\right) d x .
$$

The quality of the numerical approximation hence hinges on how well the conserved integrals can be preserved at the discrete level. The objective of this work is to develop a novel DG method to preserve these three conserved integrals at the discrete setting.

The conservation law structure of many PDEs is considered fundamental to their derivation, their behavior, and their discretization. For conservative PDEs, numerical methods preserving their invariants are often advantageous: besides the high accuracy of numerical solutions, an invariant preserving scheme can maintain good stability properties over a long-time period. Numerical methods without this property may result in substantial phase and shape errors after long time integration. There are many numerical works in the literature to solve the KdV equation, ranging from finite difference methods $[10,12,13,19,32,41]$, finite element methods $[1,29,31]$, spectral methods $[9,14,23,25,26,30]$, operator splitting methods [16, 15], and discontinuous Galerkin methods $[3,22,34,35,39]$. However, the majority of the developed methods is designed to preserve only $E_{1}$, not other invariants.

The earlier effort of inheriting some invariants as characteristic properties followed mainly the symplectic method for Hamiltonian ODEs; for instance, Furihata [10] designed a finite difference scheme to preserve both $E_{1}$ and $E_{3}$. Preservation of $E_{1}$ and $E_{2}$ in the context of the DG method has been recently investigated by Bona et al [2] and the authors [40].

Discovered by Gardner [11] and also by Faddeev and Zakharov [8], the KdV equation is a Hamiltonian system, allowing for an infinite sequence of Hamiltonians. For the generalized KdV equation (1), $E_{3}$ is still a Hamiltonian since (1) is equivalent to

$$
\partial_{t} u=\partial_{x}\left(\frac{\delta E_{3}}{\delta u}\right) \text {. }
$$

This Hamiltonian structure sets the basis to construct an $E_{3}$ preserving discretization for (1). In the case $f(u)=u^{2} / 2, E_{2}$ is also a Hamiltonian since the $\mathrm{KdV}$ equation can be written as

$$
\partial_{t} u=\mathcal{N}\left(\frac{\delta E_{2}}{\delta u}\right)
$$

where the Lenard operator $\mathcal{N}=-\left(\partial_{x}^{2}+\frac{2}{3} u \partial_{x}+\frac{1}{3} \partial_{x} u\right)$ is linear and anti-symmetric. The main novelty in this work is the preservation of $E_{3}$ by our method, which we call the Hamiltonian preserving DG method. Indeed, the proposed method is shown to preserve all three conserved integrals for the linearized $\mathrm{KdV}$ equation, and preserve both $E_{1}$ and $E_{3}$ for the generalized nonlinear $\mathrm{KdV}$ equation (1). The preservation of $E_{2}$ is investigated with care: we observe that $E_{2}$ is still preserved numerically, though its preservation is only shown in approximation sense. As illustrated in our numerical comparison (see Figure 3), the $E_{3}$-preserving scheme performs clearly better than the $E_{2}$-preserving scheme introduced in [40]. Moreover, we observe large oscillations in the temporal evolution of $E_{3}$ when testing the $E_{2}$-preserving scheme (see Figure $3(\mathrm{c})$ ). To our best knowledge, the present DG method is the first one that can preserve all three invariants $E_{i}(i=1,2,3)$ numerically.

As for the numerical convergence, we should mention that the common feature of these central fluxes based DG methods is that the optimal order of accuracy can be achieved only for polynomials of even degree, as proved for the linearized KdV equation and numerically observed for the generalized KdV equation.

The DG method we discuss in this paper is a class of finite element methods using completely discontinuous piecewise-polynomial space for the numerical solution and the test functions in the spatial variables. The application of DG methods to hyperbolic problems has been quite 
successful, e.g., Reed and Hill [28] for solving linear equations, and Cockburn et al. [4, 5, 6, 7] for solving nonlinear equations, and the DG methods have been extended to various higher order PDEs by many authors, including the KdV type equations; see, e.g., [39, 37, 2, 38, 40], the Camassa-Holm equation [36] and the Degasperis and Procesi equation [33, 21]. These DG methods have several attractive properties. For instance, it can be easily designed for any order of accuracy, with the order of accuracy locally determined in each cell, thus allowing for efficient $p$ adaptivity. The advantage of invariants preserving methods is to solve smooth wave problems, with the attempt to resolve all wave patterns for long time periods.

Obtaining a priori error estimates for various DG methods has been a main subject of research. The $L^{2}$ a priori error estimates for nonlinear PDEs with high order derivatives such as the $\mathrm{KdV}$ equations have been obtained [39, 35, 3] using certain special local projections. For the conservative DG method for the generalized KdV equation in [2], a global projection was used in obtaining error estimates in some cases. Optimal $L^{2}$ error estimates of the LDG method for the linearized KdV equation were obtained by $\mathrm{Xu}$ and Shu [38], where authors take advantage of stability estimates for auxiliary variables. The way of using error equations in the present work is closely related to that in [38].

This paper is organized as follows. In Section 2, we present the semi-discrete DG method for the generalized KdV equation, and prove its invariant preserving properties. The optimal error estimate is also analyzed for the linearized KdV equation in Section 2 when polynomials are of even degree, and therein, the upper bound of errors is proved to grow linearly in time. In Section 3, we present the fully discrete DG method with invariants preserving properties. Section 4 contains numerical experiments that demonstrate the optimal convergence rates and invariants conservation of the proposed DG method. Finally, we give the concluding remarks in Section 5 .

\section{DG FOR THE GENERALIZED KDV EQUATION}

We consider the generalized $\mathrm{KdV}$ equation of the form

$$
u_{t}=-\left(f(u)+u_{x x}\right)_{x},
$$

subject to initial data $u(x, 0)=u_{0}(x)$ and periodic boundary conditions. In order to design a DG method preserving three invariants, we reformulate this form into the following system

$$
\begin{aligned}
u_{t} & =q_{x}, \\
q & =-r_{x}-f(u), \\
r & =u_{x} .
\end{aligned}
$$

The third conservation law for the generalized KdV equation can be expressed as

$$
E_{3}=\int\left(\frac{1}{2} r^{2}-F(u)\right) d x, \quad F(u)=\int_{0}^{u} f(\xi) d \xi
$$

2.1. DG formulation. Let us denote the computational mesh of the domain $I$ by

$$
I_{j}=\left(x_{j-1 / 2}, x_{j+1 / 2}\right), \quad j=1, \ldots, N .
$$

The cell center is $x_{j}=\left(x_{j-1 / 2}+x_{j+1 / 2}\right) / 2$, with $h_{j}=x_{j+1 / 2}-x_{j-1 / 2}$, and $h=\max _{1 \leq j \leq N} h_{j}$. We assume that the mesh is regular, namely there is a constant $c>0$ independent of $h$ such that

$$
c h \leq h_{j}, \quad j=1, \cdots, N .
$$

When $c=1$, the mesh is uniform. We denote by $w_{j+1 / 2}^{+}$the value of $w$ at $x_{j+1 / 2}$ evaluated from the right element $I_{j+1}$, and $w_{j+1 / 2}^{-}$the value of $w$ at $x_{j+1 / 2}$ evaluated from the left element $I_{j}$. $[w]=w^{+}-w^{-}$denotes the jump of $w$ at cell interfaces, and $\{w\}=\frac{1}{2}\left(w^{+}+w^{-}\right)$denotes the 
average of the left and right interface values. We then define the piecewise polynomial space $V_{h}$ as the space of polynomials of degree $k$ in each cell $I_{j}$, i.e.,

$$
V_{h}=\left\{w: w \in P^{k}\left(I_{j}\right) \text { for } x \in I_{j}, j=1, \ldots, N\right\} .
$$

The DG method is formulated as follows: find $u_{h}, q_{h}, r_{h} \in V_{h}$, such that $\forall \xi, \eta, \rho \in V_{h}$,

$$
\begin{aligned}
& \int_{I_{j}} u_{h t} \xi d x=-\int_{I_{j}} q_{h} \xi_{x} d x+\left.\left(\hat{q}_{h} \xi\right)\right|_{\partial I_{j}}, \\
& \int_{I_{j}} q_{h} \eta d x=\int_{I_{j}} r_{h} \eta_{x} d x-\left.\left(\hat{r}_{h} \eta\right)\right|_{\partial I_{j}}-\int_{I_{j}} f\left(u_{h}\right) \eta d x, \\
& \int_{I_{j}} r_{h} \rho d x+\int u_{h} \rho_{x} d x-\left.\left(\hat{u}_{h} \rho\right)\right|_{\partial I_{j}}=0,
\end{aligned}
$$

with the numerical fluxes

$$
\left(\hat{q}_{h}, \hat{r}_{h}, \hat{u}_{h}\right)=\left(\left\{q_{h}\right\},\left\{r_{h}\right\},\left\{u_{h}\right\}\right) .
$$

Here we have used the notation $\left.\xi\right|_{\partial I_{j}}=\xi\left(x_{j+1 / 2}^{-}\right)-\xi\left(x_{j-1 / 2}^{+}\right)$to denote interface terms.

The global form of the DG formulation may be obtained by summing (5) over all $j$ 's,

$$
\begin{aligned}
& \int u_{h t} \xi d x=-\int q_{h} \xi_{x} d x-\sum_{j}\left(\hat{q}_{h}[\xi]\right)_{j+1 / 2} \\
& \int q_{h} \eta d x=\int r_{h} \eta_{x} d x+\sum_{j} \hat{r}_{h}[\eta]_{j+1 / 2}-\int f\left(u_{h}\right) \eta d x \\
& \int r_{h} \rho d x+\int u_{h} \rho_{x} d x+\sum_{j} \hat{u}_{h}[\rho]_{j+1 / 2}=0
\end{aligned}
$$

where $\int=\sum_{j=1}^{N} \int_{I_{j}}$, and the periodic boundary conditions have been used.

2.2. Conservative properties. We now turn to establish the conservation properties.

Theorem 2.1. Let $\left(q_{h}, r_{h}, u_{h}\right)$ be obtained from the DG scheme (7). Then both invariants $E_{1}$ and $E_{3}$ are preserved in the sense that

$$
\frac{d}{d t} \int u_{h} d x=0, \quad \frac{d}{d t} \int\left(\frac{1}{2} r_{h}^{2}-F\left(u_{h}\right)\right) d x=0 .
$$

$E_{2}$ is preserved for $f(u)=\alpha u$. For nonlinear flux, we have

$$
\frac{d}{d t} \int \frac{u_{h}^{2}}{2} d x=\sum_{j}\left(\left\{\Pi f\left(u_{h}\right)\right\}\left(u_{h}^{+}-u_{h}^{-}\right)-\int_{u_{h}^{-}}^{u_{h}^{+}} f(u) d u\right)_{x_{j+1 / 2}},
$$

where $\Pi$ denotes the piecewise $L^{2}$-projection on space $P^{k}$ :

$$
\int_{I_{j}} \Pi f(u) v d x=\int_{I_{j}} f(u) v d x, \quad \forall v \in P^{k}\left(I_{j}\right), j=1,2, \cdots, N .
$$

Proof. To see the scheme preserves $E_{1}$ we simply take $\xi=1$ in (7a) to obtain $\frac{d}{d t} \int u_{h} d x=0$. To show the conservation of $E_{3}$, we take $\xi=q_{h}$ in (7a), $\eta=-u_{h t}$ in (7b) and $\rho=r_{h}$ in $\partial_{t}(7 \mathrm{c})$ to 
obtain

$$
\begin{aligned}
& \int u_{h t} q_{h} d x=-\int q_{h} q_{h x} d x-\sum_{j}\left(\hat{q}_{h}\left[q_{h}\right]\right)_{j+1 / 2}, \\
& -\int q_{h} u_{h t} d x=-\int r_{h} u_{h t x} d x-\sum_{j} \hat{r}_{h}\left[u_{h t}\right]_{j+1 / 2}+\int f\left(u_{h}\right) u_{h t} d x \\
& \int r_{h t} r_{h} d x+\int u_{h t} r_{h x} d x+\sum_{j} \hat{u}_{h t}\left[r_{h}\right]_{j+1 / 2}=0 .
\end{aligned}
$$

Adding the above together and integrating the complete derivative out, we obtain

$$
\frac{d}{d t} \int\left(\frac{r_{h}^{2}}{2}-F\left(u_{h}\right)\right) d x=-\sum_{j=1}^{N}\left(\hat{r}_{h}\left[u_{h t}\right]+\hat{u}_{h t}\left[r_{h}\right]-\left[u_{h t} r_{h}\right]\right)_{j+1 / 2}=0
$$

We next derive temporal change rate of $E_{2}$.

Take $\xi=u_{h}, \eta=r_{h}, \rho=q_{h}$ in (7) we get

$$
\begin{aligned}
& \int u_{h t} u_{h} d x=-\int q_{h} u_{h x} d x-\sum_{j}\left(\hat{q}_{h}\left[u_{h}\right]\right)_{j+1 / 2} \\
& \int q_{h} r_{h} d x=\int r_{h} r_{h x} d x+\sum_{j} \hat{r}_{h}\left[r_{h}\right]_{j+1 / 2}-\int f\left(u_{h}\right) r_{h} d x \\
& \int r_{h} q_{h} d x+\int u_{h} q_{h x} d x+\sum_{j} \hat{u}_{h}\left[q_{h}\right]_{j+1 / 2}=0 .
\end{aligned}
$$

Adding the above together and integrating the complete derivative out, we obtain

$$
\frac{d}{d t} \int \frac{u_{h}^{2}}{2} d x=\int f\left(u_{h}\right) r_{h} d x=\sum_{j} \int_{I_{j}} \Pi f\left(u_{h}\right) r_{h} d x .
$$

Set $\rho=\Pi f\left(u_{h}\right)$ in $(7 \mathrm{c})$ we have

$$
\begin{aligned}
\frac{d}{d t} \int \frac{u_{h}^{2}}{2} d x & =\sum_{j} \int_{I_{j}} \Pi f\left(u_{h}\right) r_{h} d x \\
& =-\sum_{j} \int_{I_{j}} u_{h}\left(\Pi f\left(u_{h}\right)\right)_{x} d x-\sum_{j} \hat{u}_{h}\left[\Pi f\left(u_{h}\right)\right]_{j+1 / 2} \\
& =\sum_{j}\left(\left\{\Pi f\left(u_{h}\right)\right\}\left[u_{h}\right]\right)_{j+1 / 2}+\sum_{j} \int_{I_{j}} \Pi f\left(u_{h}\right) \cdot u_{h x} d x \\
& =\sum_{j}\left(\left\{\Pi f\left(u_{h}\right)\right\}-\frac{\int_{u_{h}^{-}}^{u_{h}^{+}} f(u) d u}{u_{h}^{+}-u_{h}^{-}}\right)\left[u_{h}\right] .
\end{aligned}
$$

Note that for linear equation $f(u)=-\alpha u$, we have

$$
\left\{\Pi f\left(u_{h}\right)\right\}-\frac{\int_{u_{h}^{-}}^{u_{h}^{+}} f(u) d u}{u_{h}^{+}-u_{h}^{-}}=0,
$$

hence $E_{2}$ is also exactly preserved. This finishes the proof of the theorem. 
Remark 2.1. For nonlinear flux, the change rate of $E_{2}$ is in general non-vanishing, but can be quite small. For instance, if $\left.u_{h}\right|_{I_{j}} \in P^{0}\left(I_{j}\right)$, the change rate reduces to

$$
\left|\sum_{j}\left(\frac{f\left(u_{h}^{+}\right)+f\left(u_{h}^{-}\right)}{2}\left[u_{h}\right]-\int_{u_{h}^{-}}^{u_{h}^{+}} f(u) d u\right)_{j+1 / 2}\right| \leq \frac{\max \left|f^{\prime \prime}\right|}{12} \sum_{j}\left|u_{h}^{+}-u_{h}^{-}\right|_{j+1 / 2}^{3} \sim h^{2},
$$

at least for approximating smooth solutions.

Remark 2.2. In the DG formulation (7), the nonlinear term $\int f\left(u_{h}\right) \eta d x$ is calculated by quadratures with accuracy of order bigger than $O\left(h^{k+2}\right)$. For instance, if we use a Gauss quadrature with $L \geq \frac{k}{2}+1$ points, it has accuracy of order at least $O\left(h^{k+2}\right)$. The evaluation of $E_{3}$ involves the calculation of $\int F\left(u_{h}\right) d x$, for which we need to use quadratures with sufficient accuracy. In our numerical examples, $f(u)=u^{2} / 2$, then $F\left(u_{h}\right)$ is a polynomial of degree $3 k$, for which we use a Gauss quadrature with $L$ points, $L \geq \frac{3 k-1}{2}$, so that the numerical evaluation of $E_{3}$ is exact.

2.3. Error estimation for the linearized $\mathbf{K d V}$ equation. In the following, we derive the error estimation of the discontinuous Galerkin method for the linearized KdV equation of the form

$$
u_{t}-\alpha u_{x}+u_{x x x}=0,
$$

subject to initial data $u_{0}(x)$ and periodic boundary conditions.

The corresponding DG method for (10) is formulated as follows: find $u_{h}, q_{h}, r_{h} \in V_{h}$, such that $\forall \xi, \eta, \rho \in V_{h}$,

$$
\begin{aligned}
& \int_{I_{j}} u_{h t} \xi d x+\int_{I_{j}} q_{h} \xi_{x} d x-\left.\hat{q}_{h} \xi\right|_{\partial I_{j}}=0, \\
& \int_{I_{j}} q_{h} \eta d x-\int_{I_{j}} r_{h} \eta_{x} d x+\left.\hat{r}_{h} \eta\right|_{\partial I_{j}}-\alpha \int_{I_{j}} u_{h} \eta d x=0, \\
& \int_{I_{j}} r_{h} \rho d x+\int u_{h} \rho_{x} d x-\left.\hat{u}_{h} \rho\right|_{\partial I_{j}}=0 .
\end{aligned}
$$

with the central numerical fluxes

$$
\left(\hat{q}_{h}, \hat{r}_{h}, \hat{u}_{h}\right)=\left(\left\{q_{h}\right\},\left\{r_{h}\right\},\left\{u_{h}\right\}\right) .
$$

For the error estimates, we introduce a special global projection $P$ as follows. For any $u$, $\left.P u\right|_{I_{j}} \in P^{k}\left(I_{j}\right)$ satisfies

$$
\begin{gathered}
(P u, v)_{I_{j}}=(u, v)_{I_{j}}, \quad \forall v \in P^{k-1}\left(I_{j}\right), \\
\left.\{P u\}\right|_{x_{j+\frac{1}{2}}}=u\left(x_{j+\frac{1}{2}}\right), \quad j=1,2, \cdots, N .
\end{gathered}
$$

Lemma 2.2. Suppose the mesh is regular, and $u$ is sufficiently smooth and periodic. Assume that $k$ is even and that the number of cells $N$ is odd. Then the projection $P$ defined in (13) exists, and has the approximation property,

$$
\|u-P u\|_{L^{2}(I)} \leq C h^{k+1},
$$

for a constant $C$ independent of the mesh size $h$.

Proof. Step 1. We first show the existence of such a projection. Let $\left\{L_{i}(\xi)\right\}_{i=0}^{k}$ be the Legendre polynomials on $[-1,1]$ with $\left\|L_{i}(\xi)\right\|_{L^{2}(\hat{I})}^{2}=\frac{2}{2 i+1}$, and $\phi_{i}^{j}(x)=L_{i}\left(2\left(x-x_{j}\right) / h_{j}\right)$, then the projection of $u$ can be expressed as

$$
\left.P u(x)\right|_{I_{j}}=\sum_{i=0}^{k} u_{i}^{j} \phi_{i}^{j}(x), \quad 1 \leq j \leq N .
$$


From (13a) with $v=\left\{\phi_{i}^{j}\right\}_{i=0}^{k-1}$, it follows that for $1 \leq j \leq N$,

$$
u_{i}^{j}=\frac{2 i+1}{2} \int_{\hat{I}} \hat{u}^{j}(\xi) L_{i}(\xi) d \xi, \quad i=0,1, \cdots, k-1,
$$

where $\hat{u}^{j}(\xi)=u\left(x_{j}+\frac{h_{j}}{2} \xi\right)$. It remains to determine $u_{k}^{j}$ by using the interface conditions (13b), which leads to

$$
u_{k}^{j}+(-1)^{k} u_{k}^{j+1}=b_{j}:=2 u\left(x_{j+1 / 2}\right)-\sum_{i=0}^{k-1}\left(u_{i}^{j}+(-1)^{j} u_{i}^{j+1}\right), \quad j=1,2, \cdots, N .
$$

The above equations can be written in matrix form as follows,

$$
\left[\begin{array}{cccc}
1 & (-1)^{k} & & \\
& 1 & \ddots & \\
& & \ddots & (-1)^{k} \\
(-1)^{k} & & & 1
\end{array}\right]\left[\begin{array}{c}
u_{k}^{1} \\
u_{k}^{2} \\
\vdots \\
u_{k}^{N}
\end{array}\right]=\left[\begin{array}{c}
b_{1} \\
b_{2} \\
\vdots \\
b_{N} \cdot
\end{array}\right]
$$

It is clear that the determinant of coefficient matrix of (15) is nonzero if and only if $k$ is even and $N$ is odd. Then the existence of global projection (13) follows.

Step 2. We now show the projection error. For $i=0,1, \cdots, k-1, j=1,2, \cdots, N$, we have

$$
\left|u_{i}^{j}\right|^{2}=\frac{(2 i+1)^{2}}{4}\left(\int_{\hat{I}} \hat{u}^{j}(\xi) L_{i}(\xi) d \xi\right)^{2} \leq \frac{2 i+1}{2}\left\|\hat{u}^{j}(\cdot)\right\|_{L^{2}(\hat{I})}^{2} .
$$

For $\left\{u_{k}^{j}\right\}_{j=1}^{N}$, from (15) and (16) we have

$$
\begin{aligned}
\sum_{j=1}^{N}\left(u_{k}^{j}\right)^{2} & \leq C \sum_{j=1}^{N}\left|b_{j}\right|^{2} \leq C \sum_{j=1}^{N}\left(\left|u\left(x_{j+1 / 2}\right)\right|^{2}+\sum_{i=0}^{k-1}\left|u_{i}^{j}\right|^{2}\right) \\
& \leq C \sum_{j=1}^{N}\left(\left\|\hat{u}^{j}(\xi)\right\|_{L^{\infty}(\hat{I})}^{2}+k^{2}\left\|\hat{u}^{j}(\cdot)\right\|_{L^{2}(\hat{I})}^{2}\right) \leq C \sum_{j=1}^{N}\left\|\hat{u}^{j}(\cdot)\right\|_{H^{1}(\hat{I})}^{2}
\end{aligned}
$$

From (16) and (17), we obtain

$$
\begin{aligned}
\sum_{j=1}^{N} \sum_{i=0}^{k}\left|u_{i}^{j}\right|^{2} & =\sum_{j=1}^{N} \sum_{i=0}^{k-1}\left|u_{i}^{j}\right|^{2}+\sum_{j=1}^{N}\left|u_{k}^{j}\right|^{2} \\
& \leq \frac{k^{2}}{2} \sum_{j=1}^{N}\left\|\hat{u}^{j}(\cdot)\right\|_{L^{2}(\hat{I})}^{2}+C \sum_{j=1}^{N}\left\|\hat{u}^{j}(\cdot)\right\|_{H^{1}(\hat{I})}^{2} \\
& \leq C \sum_{j=1}^{N}\left\|\hat{u}^{j}(\cdot)\right\|_{H^{1}(\hat{I})}^{2} .
\end{aligned}
$$

Then we get the following stability property for the projection $P$ :

$$
\begin{aligned}
\|P u\|_{L^{2}(I)}^{2} & =\sum_{j=1}^{N} \frac{h_{j}}{2} \int_{\hat{I}}\left(\sum_{i=0}^{k} u_{i}^{j} L_{i}(\xi)\right)^{2} d \xi \\
& \leq h \sum_{j=1}^{N} \sum_{i=0}^{k}\left(u_{i}^{j}\right)^{2} \leq C h \sum_{j=1}^{N}\left\|\hat{u}^{j}(\cdot)\right\|_{H^{1}(\hat{I})}^{2}
\end{aligned}
$$


Using (19) and (18), we derive the $L^{2}$-error estimation (14) as follows.

$$
\begin{aligned}
\|u-P u\|_{L^{2}(I)}^{2} & =\inf _{v \in V_{h}}\|(I-P)(u+v)\|_{L^{2}(I)}^{2} \leq C h \sum_{j=1}^{N} \inf _{\hat{v} \in P^{k}(\hat{I})}\left\|\hat{u}^{j}+\hat{v}\right\|_{H^{1}(\hat{I})}^{2} \\
& \leq C h \sum_{j=1}^{N}\left|\hat{u}^{j}\right|_{H^{k+1}(\hat{I})}^{2}=C h \sum_{j=1}^{N}\left(\frac{h_{j}}{2}\right)^{2 k+1}|u|_{H^{k+1}\left(I_{j}\right)}^{2} \\
& \leq C h^{2 k+2}|u|_{H^{k+1}(I)}^{2} .
\end{aligned}
$$

In order to obtain the error estimate to smooth solutions for the semi-discrete DG scheme (11), we first write the global form obtained by summing over all $j$ 's in (11),

$$
\begin{aligned}
& \left(u_{h t}, \xi\right)+\left(q_{h}, \xi_{x}\right)+\sum_{j}\left(\hat{q}_{h}[\xi]\right)_{j+1 / 2}=0, \\
& \left(q_{h}, \eta\right)-\left(r_{h}, \eta_{x}\right)-\sum_{j}\left(\hat{r}_{h}[\eta]\right)_{j+1 / 2}-\alpha\left(u_{h}, \eta\right)=0, \\
& \left(r_{h}, \rho\right)+\left(u_{h}, \rho_{x}\right)+\sum_{j}\left(\hat{u}_{h}[\rho]\right)_{j+1 / 2}=0,
\end{aligned}
$$

where the periodic boundary conditions have been used. The consistency of the DG scheme implies that the exact solution $u$, with $r=u_{x}, q=-u_{x x}+\alpha u$ also satisfies (20). Hence for

$$
\left(e_{u}, e_{q}, e_{r}\right)=\left(u-u_{h}, q-q_{h}, r-r_{h}\right),
$$

we have the following error equations:

$$
\begin{aligned}
& \left(\left(e_{u}\right)_{t}, \xi\right)+\left(e_{q}, \xi_{x}\right)+\sum_{j}\left(\widehat{e_{q}}[\xi]\right)_{j+1 / 2}=0, \\
& \left(e_{q}, \eta\right)-\left(e_{r}, \eta_{x}\right)-\sum_{j}\left(\widehat{e_{r}}[\eta]\right)_{j+1 / 2}-\alpha\left(e_{u}, \eta\right)=0, \\
& \left(e_{r}, \rho\right)+\left(e_{u}, \rho_{x}\right)-\sum_{j}\left(\widehat{e_{u}}[\rho]\right)_{j+1 / 2}=0 .
\end{aligned}
$$

Let $P$ be the global projection defined in (13), we use the following decomposition:

$$
\begin{aligned}
& e_{u}=(u-P u)+\left(P u-u_{h}\right)=e_{u}^{P}+e_{u}^{D}, \\
& e_{q}=(q-P q)+\left(P q-q_{h}\right)=e_{q}^{P}+e_{q}^{D}, \\
& e_{r}=(r-P r)+\left(P r-r_{h}\right)=e_{r}^{P}+e_{r}^{D} .
\end{aligned}
$$

First we have the following.

Lemma 2.3. Let $(u, q, r)$ and $\left(u_{h}, q_{h}, r_{h}\right)$ be the exact solutions and their numerical approximations, respectively, then

$$
\left(e_{r}, e_{u}^{D}\right)=0, \quad\left(\left(e_{r}\right)_{t},\left(e_{u}^{D}\right)_{t}\right)=0
$$

Proof. Take $\rho=e_{u}^{D}$ in equation (21c) to get

$$
\left(e_{r}, e_{u}^{D}\right)+\left(e_{u}^{P},\left(e_{u}^{D}\right)_{x}\right)+\left(e_{u}^{D},\left(e_{u}^{D}\right)_{x}\right)+\sum_{j}\left(\widehat{e_{u}^{P}}\left[e_{u}^{D}\right]\right)_{j+1 / 2}+\sum_{j}\left(\widehat{e_{u}^{D}}\left[e_{u}^{D}\right]\right)_{j+1 / 2}=0 .
$$


With

$$
\left(e_{u}^{D},\left(e_{u}^{D}\right)_{x}\right)+\sum_{j}\left(\widehat{e_{u}^{D}}\left[e_{u}^{D}\right]\right)_{j+1 / 2}=0
$$

and the definition of the global projection (13), we have

$$
\left(e_{r}, e_{u}^{D}\right)=0 .
$$

In a similar manner, we take the time derivative in (21c), and choose $\rho=\left(e_{u}^{D}\right)_{t}$, to get

$$
\left(\left(e_{r}\right)_{t},\left(e_{u}^{D}\right)_{t}\right)=0 \text {. }
$$

We proceed to derive the energy equations for $e_{u}, e_{q}$ and $e_{r}$, from which the error estimates follow. Take the test functions $\xi=e_{u}^{D}, \eta=-e_{r}^{D}$, and $\rho=e_{q}^{D}$ in (21) to get

$$
\begin{aligned}
& \left(\left(e_{u}\right)_{t}, e_{u}^{D}\right)+\left(e_{q},\left(e_{u}^{D}\right)_{x}\right)+\sum_{j}\left(\widehat{e_{q}}\left[e_{u}^{D}\right]\right)_{j+1 / 2}=0, \\
& -\left(e_{q}, e_{r}^{D}\right)+\left(e_{r},\left(e_{r}^{D}\right)_{x}\right)+\sum_{j}\left(\widehat{e_{r}}\left[e_{r}^{D}\right]\right)_{j+1 / 2}+\alpha\left(e_{u}, e_{r}^{D}\right)=0, \\
& \left(e_{r}, e_{q}^{D}\right)+\left(e_{u},\left(e_{q}^{D}\right)_{x}\right)+\sum_{j}\left(\widehat{e_{u}}\left[e_{q}^{D}\right]\right)_{j+1 / 2}=0 .
\end{aligned}
$$

These in virtue of decomposition (22) and the projection properties yield

$$
\begin{aligned}
& \left(\left(e_{u}\right)_{t}, e_{u}^{D}\right)+\left(e_{q}^{D},\left(e_{u}^{D}\right)_{x}\right)+\sum_{j}\left(\widehat{e_{q}^{D}}\left[e_{u}^{D}\right]\right)_{j+1 / 2}=0, \\
& \alpha\left(e_{u}, e_{r}^{D}\right)-\left(e_{q}^{P}, e_{r}^{D}\right)-\left(e_{q}^{D}, e_{r}^{D}\right)=0, \\
& \left(e_{u}^{D},\left(e_{q}^{D}\right)_{x}\right)+\sum_{j}\left(\widehat{e_{u}^{D}}\left[e_{q}^{D}\right]\right)_{j+1 / 2}+\left(e_{r}, e_{q}^{D}\right)=0 .
\end{aligned}
$$

In the second equation we have used $\left(e_{r}^{D},\left(e_{r}^{D}\right)_{x}\right)+\sum_{j}\left(\widehat{e_{r}^{D}}\left[e_{r}^{D}\right]\right)_{j+1 / 2}=0$. Note that

$$
\left(e_{q}^{D},\left(e_{u}^{D}\right)_{x}\right)+\left(e_{u}^{D},\left(e_{q}^{D}\right)_{x}\right)+\sum_{j}\left(\widehat{e_{q}^{D}}\left[e_{u}^{D}\right]\right)_{j+1 / 2}+\sum_{j}\left(\widehat{e_{u}^{D}}\left[e_{q}^{D}\right]\right)_{j+1 / 2}=0
$$

so that the sum of all three equations in (27) gives

$$
\left(\left(e_{u}\right)_{t}, e_{u}^{D}\right)+\alpha\left(e_{u}, e_{r}^{D}\right)-\left(e_{q}^{P}, e_{r}^{D}\right)+\left(e_{r}^{P}, e_{q}^{D}\right)=0 .
$$

From the first equation of $(23)$, we have $\left(e_{u}^{D}, e_{r}^{D}\right)+\left(e_{u}^{D}, e_{r}^{P}\right)=0$. Hence (28) reduces to

$$
\left(\left(e_{u}^{P}\right)_{t}, e_{u}^{D}\right)+\left(\left(e_{u}^{D}\right)_{t}, e_{u}^{D}\right)+\alpha\left(e_{u}^{P}, e_{r}^{D}\right)-\alpha\left(e_{u}^{D}, e_{r}^{P}\right)-\left(e_{q}^{P}, e_{r}^{D}\right)+\left(e_{r}^{P}, e_{q}^{D}\right)=0 .
$$

We take the time derivative in (21b)-(21c), with (21a), to obtain

$$
\begin{aligned}
& \left(\left(e_{u}\right)_{t}, \xi\right)+\left(e_{q}, \xi_{x}\right)+\sum_{j}\left(\widehat{e_{q}}[\xi]\right)_{j+1 / 2}=0, \\
& -\alpha\left(\left(e_{u}\right)_{t}, \eta\right)+\left(\left(e_{q}\right)_{t}, \eta\right)-\left(\left(e_{r}\right)_{t}, \eta_{x}\right)-\sum_{j}\left(\widehat{\left(e_{r}\right)_{t}}[\eta]\right)_{j+1 / 2}=0, \\
& \left(\left(e_{u}\right)_{t}, \rho_{x}\right)+\sum_{j}\left(\widehat{\left(e_{u}\right)_{t}}[\rho]\right)_{j+1 / 2}+\left(\left(e_{r}\right)_{t}, \rho\right)=0 .
\end{aligned}
$$


Taking the test functions $\xi=-\left(e_{r}^{D}\right)_{t}, \eta=e_{q}^{D}$, and $\rho=\left(e_{u}^{D}\right)_{t}$ in (30), we get

$$
\begin{aligned}
& -\left(\left(e_{u}\right)_{t},\left(e_{r}^{D}\right)_{t}\right)-\left(e_{q},\left(\left(e_{r}^{D}\right)_{t x}\right)-\sum_{j}\left(\widehat{e_{q}}\left[\left(e_{r}^{D}\right)_{t}\right]\right)_{j+1 / 2}=0,\right. \\
& -\alpha\left(\left(e_{u}\right)_{t}, e_{q}^{D}\right)+\left(\left(e_{q}\right)_{t}, e_{q}^{D}\right)-\left(\left(e_{r}\right)_{t},\left(e_{q}^{D}\right)_{x}\right)-\sum_{j}\left(\widehat{\left(e_{r}\right)_{t}}\left[e_{q}^{D}\right]\right)_{j+1 / 2}=0, \\
& \left(\left(e_{u}\right)_{t},\left(e_{u}^{D}\right)_{t x}\right)+\sum_{j}\left(\widehat{\left(e_{u}\right)_{t}}\left[\left(e_{u}^{D}\right)_{t}\right]\right)_{j+1 / 2}+\left(\left(e_{r}\right)_{t},\left(e_{u}^{D}\right)_{t}\right)=0 .
\end{aligned}
$$

Using the notation in (22) and the projection properties, we have

$$
\begin{aligned}
& -\left(\left(e_{u}\right)_{t},\left(e_{r}^{D}\right)_{t}\right)-\left(e_{q}^{D},\left(\left(e_{r}^{D}\right)_{t x}\right)-\sum_{j}\left(\widehat{e_{q}^{D}}\left[\left(e_{r}^{D}\right)_{t}\right]\right)_{j+1 / 2}=0,\right. \\
& -\alpha\left(\left(e_{u}\right)_{t}, e_{q}^{D}\right)+\left(\left(e_{q}\right)_{t}, e_{q}^{D}\right)-\left(\left(e_{r}^{D}\right)_{t},\left(e_{q}^{D}\right)_{x}\right)-\sum_{j}\left(\widehat{\left(e_{r}^{D}\right)_{t}}\left[e_{q}^{D}\right]\right)_{j+1 / 2}=0, \\
& \left(\left(e_{u}^{D}\right)_{t},\left(e_{u}^{D}\right)_{t x}\right)+\left(\left(e_{r}^{P}\right)_{t},\left(e_{u}^{D}\right)_{t}\right)+\left(\left(e_{r}^{D}\right)_{t},\left(e_{u}^{D}\right)_{t}\right)+\sum_{j}\left(\widehat{\left(e_{u}^{D}\right)_{t}}\left[\left(e_{u}^{D}\right)_{t}\right]\right)_{j+1 / 2}=0 .
\end{aligned}
$$

Note that

$$
\begin{array}{r}
\left(\left(e_{u}^{D}\right)_{t},\left(e_{u}^{D}\right)_{t x}\right)+\sum_{j}\left(\widehat{\left(e_{u}^{D}\right)_{t}}\left[\left(e_{u}^{D}\right)_{t}\right]\right)_{j+1 / 2}=0, \\
\left(e_{q}^{D},\left(e_{r}^{D}\right)_{t x}\right)+\left(e_{r}^{D},\left(e_{q}^{D}\right)_{t x}\right)+\sum_{j}\left(\widehat{e_{q}^{D}}\left[\left(e_{r}^{D}\right)_{t}\right]\right)_{j+1 / 2}+\sum_{j}\left(\widehat{\left(e_{r}^{D}\right)_{t}}\left[e_{q}^{D}\right]\right)_{j+1 / 2}=0,
\end{array}
$$

so that the sum of all three equations in (32) leads to

(33) $\left(\left(e_{q}^{P}\right)_{t}, e_{q}^{D}\right)+\left(\left(e_{q}^{D}\right)_{t}, e_{q}^{D}\right)-\left(\left(e_{u}^{P}\right)_{t},\left(e_{r}^{D}\right)_{t}\right)-\alpha\left(\left(e_{u}^{P}\right)_{t}, e_{q}^{D}\right)-\alpha\left(\left(e_{u}^{D}\right)_{t}, e_{q}^{D}\right)+\left(\left(e_{r}^{P}\right)_{t},\left(e_{u}^{D}\right)_{t}\right)=0$.

We take the time derivative in (21c), with (21b), to obtain

$$
\begin{aligned}
& -\alpha\left(e_{u}, \eta\right)+\left(e_{q}, \eta\right)-\left(e_{r}, \eta_{x}\right)-\sum_{j}\left(\widehat{\left(e_{r}\right)}[\eta]\right)_{j+1 / 2}=0, \\
& \left(\left(e_{u}\right)_{t}, \rho_{x}\right)+\sum_{j}\left(\widehat{\left(e_{u}\right)_{t}}[\rho]\right)_{j+1 / 2}+\left(\left(e_{r}\right)_{t}, \rho\right)=0 .
\end{aligned}
$$

Taking the test functions $\eta=-\left(e_{u}^{D}\right)_{t}$, and $\rho=e_{r}^{D}$ in (34), we get

$$
\begin{aligned}
& \alpha\left(e_{u},\left(e_{u}^{D}\right)_{t}\right)-\left(e_{q},\left(e_{u}^{D}\right)_{t}\right)+\left(e_{r},\left(\left(e_{u}^{D}\right)_{t x}\right)+\sum_{j}\left(\widehat{e}_{r}\left[\left(e_{u}^{D}\right)_{t}\right]\right)_{j+1 / 2}=0,\right. \\
& \left(\left(e_{u}\right)_{t},\left(e_{r}^{D}\right)_{x}\right)+\sum_{j}\left(\widehat{\left(e_{u}\right)_{t}}\left[e_{r}^{D}\right]\right)_{j+1 / 2}+\left(\left(e_{r}\right)_{t}, e_{r}^{D}\right)=0 .
\end{aligned}
$$

Using the notation in (22) and the projection properties, we have

$$
\begin{aligned}
& \alpha\left(e_{u},\left(e_{u}^{D}\right)_{t}\right)-\left(e_{q},\left(e_{u}^{D}\right)_{t}\right)+\left(e_{r}^{D},\left(e_{u}^{D}\right)_{t x}\right)+\sum_{j}\left(\widehat{e_{r}^{D}}\left[\left(e_{u}^{D}\right)_{t}\right]\right)_{j+1 / 2}=0 \\
& \left(\left(e_{u}^{D}\right)_{t},\left(e_{r}^{D}\right)_{x}\right)+\left(\left(e_{r}\right)_{t}, e_{r}^{D}\right)+\sum_{j}\left(\widehat{\left(e_{u}^{D}\right)_{t}}\left[e_{r}^{D}\right]\right)_{j+1 / 2}=0 .
\end{aligned}
$$

Note that

$$
\left(e_{r}^{D},\left(e_{u}^{D}\right)_{t x}\right)+\left(\left(e_{u}^{D}\right)_{t},\left(e_{r}^{D}\right)_{x}\right)+\sum_{j}\left(\widehat{e_{r}^{D}}\left[\left(e_{u}^{D}\right)_{t}\right]\right)_{j+1 / 2}+\sum_{j}\left(\widehat{\left(e_{u}^{D}\right)_{t}}\left[e_{r}^{D}\right]\right)_{j+1 / 2}=0
$$


then adding two equations in (36) gives

$$
\alpha\left(e_{u}^{P},\left(e_{u}^{D}\right)_{t}\right)+\alpha\left(e_{u}^{D},\left(e_{u}^{D}\right)_{t}\right)+\left(\left(e_{r}^{P}\right)_{t}, e_{r}^{D}\right)+\left(\left(e_{r}^{D}\right)_{t}, e_{r}^{D}\right)-\left(e_{q}^{P},\left(e_{u}^{D}\right)_{t}\right)-\left(e_{q}^{D},\left(e_{u}^{D}\right)_{t}\right)=0 .
$$

Similar in deriving the equation (29), we take the time derivative in (21c) and choose the test functions $\xi=\left(e_{u}^{D}\right)_{t}, \eta=-\left(e_{r}^{D}\right)_{t}$, and $\rho=\left(e_{q}^{D}\right)_{t}$. After some algebraic manipulation, we get

$\left(\left(e_{u}^{P}\right)_{t t},\left(e_{u}^{D}\right)_{t}\right)+\left(\left(e_{u}^{D}\right)_{t t},\left(e_{u}^{D}\right)_{t}\right)+\alpha\left(\left(e_{u}^{P}\right)_{t},\left(e_{r}^{D}\right)_{t}\right)+\alpha\left(\left(e_{u}^{D}\right)_{t},\left(e_{r}^{D}\right)_{t}\right)-\left(\left(e_{q}^{P}\right)_{t},\left(e_{r}^{D}\right)_{t}\right)+\left(\left(e_{r}^{P}\right)_{t},\left(e_{q}^{D}\right)_{t}\right)=0$.

From the second equation of (23), we have

$$
\left(\left(e_{u}^{D}\right)_{t},\left(e_{r}^{D}\right)_{t}\right)+\left(\left(e_{u}^{D}\right)_{t},\left(e_{r}^{P}\right)_{t}\right)=0 .
$$

Then we get

$\left(\left(e_{u}^{P}\right)_{t t},\left(e_{u}^{D}\right)_{t}\right)+\left(\left(e_{u}^{D}\right)_{t t},\left(e_{u}^{D}\right)_{t}\right)+\alpha\left(\left(e_{u}^{P}\right)_{t},\left(e_{r}^{D}\right)_{t}\right)-\alpha\left(\left(e_{u}^{D}\right)_{t},\left(e_{r}^{P}\right)_{t}\right)-\left(\left(e_{q}^{P}\right)_{t},\left(e_{r}^{D}\right)_{t}\right)+\left(\left(e_{r}^{P}\right)_{t},\left(e_{q}^{D}\right)_{t}\right)=0$.

Summing all the four equations (29), (33), (37) and (39) together, after some algebraic manipulation, we obtain

$$
(1+\alpha)\left(\left(e_{u}^{D}\right)_{t}, e_{u}^{D}\right)+\left(\left(e_{q}^{D}\right)_{t}, e_{q}^{D}\right)+\left(\left(e_{r}^{D}\right)_{t}, e_{r}^{D}\right)+\left(\left(e_{u}^{D}\right)_{t t},\left(e_{u}^{D}\right)_{t}\right)+I_{P}+I_{D}=0,
$$

where

$$
\begin{aligned}
I_{P}= & \left(\left(e_{u}^{P}\right)_{t}, e_{u}^{D}\right)+\alpha\left(e_{u}^{P}, e_{r}^{D}\right)-\alpha\left(e_{r}^{P}, e_{u}^{D}\right)-\left(e_{q}^{P}, e_{r}^{D}\right)+\left(e_{r}^{P}, e_{q}^{D}\right) \\
& +\left(\left(e_{q}^{P}\right)_{t}, e_{q}^{D}\right)-\alpha\left(\left(e_{u}^{P}\right)_{t}, e_{q}^{D}\right)+(\alpha-1)\left(\left(e_{u}^{P}\right)_{t},\left(e_{r}^{D}\right)_{t}\right)+(1-\alpha)\left(\left(e_{r}^{P}\right)_{t},\left(e_{u}^{D}\right)_{t}\right) \\
& +\alpha\left(e_{u}^{P},\left(e_{u}^{D}\right)_{t}\right)+\left(\left(e_{r}^{P}\right)_{t}, e_{r}^{D}\right)-\left(\left(e_{q}^{P},\left(e_{u}^{D}\right)_{t}\right)\right. \\
& +\left(\left(e_{u}^{P}\right)_{t t},\left(e_{u}^{D}\right)_{t}\right)-\left(\left(e_{q}^{P}\right)_{t},\left(e_{r}^{D}\right)_{t}\right)+\left(\left(e_{r}^{P}\right)_{t},\left(e_{q}^{D}\right)_{t}\right)
\end{aligned}
$$

and

$$
I_{D}=-(1+\alpha)\left(\left(e_{u}^{D}\right)_{t}, e_{q}^{D}\right) .
$$

Integration of (40) with respect to time between 0 and $t$ gives

$$
\begin{aligned}
\frac{1+\alpha}{2}\left\|e_{u}^{D}(t)\right\|^{2} & +\frac{1}{2}\left\|e_{q}^{D}(t)\right\|^{2}+\frac{1}{2}\left\|e_{r}^{D}(t)\right\|^{2}+\frac{1}{2}\left\|\left(e_{u}^{D}(t)\right)_{t}\right\|^{2} \\
& =\frac{1+\alpha}{2}\left\|e_{u}^{D}(0)\right\|^{2}+\frac{1}{2}\left\|e_{q}^{D}(0)\right\|^{2}+\frac{1}{2}\left\|e_{r}^{D}(0)\right\|^{2}+\frac{1}{2}\left\|\left(e_{u}^{D}(0)\right)_{t}\right\|^{2}-\int_{0}^{t}\left(I_{P}+I_{D}\right) d \tau .
\end{aligned}
$$

We now estimate the terms in $\int_{0}^{t} I_{P} d t$ and $\int_{0}^{t} I_{D} d t$, respectively. Using the approximation property of the projection in (14), we have

$$
\left\|\partial_{t}^{i}\left(e_{\phi}^{P}\right)\right\| \leq C h^{k+1}, \quad 0 \leq i \leq 2
$$

for $\phi \in\{u, q, r\}$. Upon further integration by parts in time for 3 out of 15 terms in $I_{P}$ and further using the Cauchy-Schwartz inequality, we have

$$
\begin{aligned}
-\int_{0}^{t} I_{P} d \tau \leq & \int_{0}^{t} C h^{k+1}\left(\left\|e_{u}^{D}(t)\right\|+\left\|e_{q}^{D}(\tau)\right\|+\left\|e_{r}^{D}(\tau)\right\|+\left\|\left(e_{u}^{D}(\tau)\right)_{t}\right\|\right) d \tau \\
& \left.+\left[(\alpha-1)\left(\left(e_{u}^{P}\right)_{t}, e_{r}^{D}\right)-\left(\left(e_{q}^{P}\right)_{t}, e_{r}^{D}\right)\right)+\left(\left(e_{r}^{P}\right)_{t}, e_{q}^{D}\right)\right]\left.\right|_{0} ^{t} \\
\leq & C h^{2 k+2}+\int_{0}^{t} F(\tau) d \tau+\frac{1}{4}\left[\left\|e_{q}^{D}(t)\right\|^{2}+\left\|e_{r}^{D}(t)\right\|^{2}\right)+\left(\left\|e_{q}^{D}(0)\right\|^{2}+\left\|e_{r}^{D}(0)\right\|^{2}\right],
\end{aligned}
$$

where $F(t)=\left\|e_{u}^{D}(t)\right\|^{2}+\left\|e_{q}^{D}(t)\right\|^{2}+\left\|e_{r}^{D}(t)\right\|^{2}+\left\|\left(e_{u}^{D}(t)\right)_{t}\right\|^{2}$. And

$$
-\int_{0}^{t} I_{D} d \tau \leq \frac{1+\alpha}{2} \int_{0}^{t} F(\tau)^{2} d \tau
$$


By the Grownwall inequality, we obtain

$$
F(t) \leq C F(0)+C h^{2 k+2} .
$$

Similar as in [17], we choose the initial condition such that

$$
q_{h}(x, 0)=P q(x, 0), \quad q(x, 0)=-u_{0 x x}(x)+\alpha u_{0}(x) .
$$

From $(11 \mathrm{~b})$ we find $r_{h}(x, 0)$ and further from $(11 \mathrm{c})$ we find $u_{h}(x, 0)$ so that they satisfy (see [38]) (43)

$\left\|u(x, 0)-u_{h}(x, 0)\right\|+\left\|q(x, 0)-q_{h}(x, 0)\right\|+\left\|r(x, 0)-r_{h}(x, 0)\right\|+\left\|u_{t}(x, 0)-\left(u_{h}\right)_{t}(x, 0)\right\| \leq C h^{k+1}$.

The triangle inequality with the estimate (43) and (14) implies $F(0) \leq C h^{2 k+2}$; hence $F \leq$ $C h^{2 k+2}$, which using (14) again implies the following result.

Theorem 2.4. Assume that the linear $K d V$ equation (10) with initial data and periodic boundary conditions admits a smooth solution $u$. Let $(u, q, r)=\left(u,-u_{x x}+\alpha u, u_{x}\right)$, and $\left(u_{h}, q_{h}, r_{h}\right)$ be the numerical approximation. Then

$$
\left\|\left(u-u_{h}\right)(t)\right\|+\left\|\left(q-q_{h}\right)(t)\right\|+\left\|\left(r-r_{h}\right)(t)\right\|+\left\|\left(u-u_{h}\right)_{t}(t)\right\| \leq C h^{k+1}
$$

holds for $k$ even and $N$ odd.

Remark 2.3. The error estimate for nonlinear $f(u)$ appears difficult with the above analysis, since the scheme has no extra numerical dissipation for controlling the trouble terms from the nonlinear effect.

\section{TIME DISCRETIZATION}

We now turn to time discretization of (7). Let $\left\{t^{n}\right\}, n=0,1, \ldots, M$ be a uniform partition of the time interval $[0, T]$. Let $U^{0}=\Pi U_{0}$ be the piecewise $L^{2}$ projection of $U_{0}(x)$.

3.1. Algorithm. We first give details related to the implementation of the method.

1. First, from (7c), we obtain $r$ in the following matrix form

$$
r_{h}=A u_{h},
$$

where $U_{h}$ denotes the vector containing the degree of freedom for $U$.

2. From (7b) we obtain

$$
q_{h}=-A r_{h}-\left(\Pi\left(f\left(u_{h}\right)\right)\right)_{h},
$$

where $\Pi$ is the piecewise $L^{2}$ projection on $V_{h}$.

3. We then substitute (45) and (46) into (7a) to obtain

$$
\partial_{t} u_{h}=A q_{h}
$$

4. We use a time discretization method to solve the obtained semi-discrete system (47).

The differential matrix $A$ is a sparse block matrix, hence its multiplication with vectors involving it as the coefficient matrix, can be implemented efficiently.

In our numerical simulation, we use a semi-implicit time discretization so that two quantities $E_{1}$ and $E_{3}$ are remain conserved in time.

We define the following operator

$$
D_{t} u_{h}^{n}=\frac{u_{h}^{n+1}-u_{h}^{n}}{\Delta t}, \quad A u_{h}^{n}=\frac{u_{h}^{n+1}+u_{h}^{n}}{2},
$$


so that the fully discrete scheme has the form

$$
\begin{aligned}
& \int D_{t} u_{h}^{n} \xi d x=-\int A q_{h}^{n} \xi_{x} d x-\sum_{j}\left(\widehat{A q_{h}^{n}}[\xi]\right)_{j+1 / 2}, \\
& \int A q_{h}^{n} \eta d x=\int A r_{h}^{n} \eta_{x} d x+\sum_{j} \widehat{A r_{h}^{n}}[\eta]_{j+1 / 2}-\int \frac{F\left(u_{h}^{n+1}\right)-F\left(u_{h}^{n}\right)}{u_{h}^{n+1}-u_{h}^{n}} \eta d x, \\
& \int r_{h}^{n} \rho d x+\int u_{h}^{n} \rho_{x} d x+\sum_{j} \widehat{u_{h}^{n}}[\rho]_{j+1 / 2}=0,
\end{aligned}
$$

where $\int=\sum_{j=1}^{N} \int_{I_{j}}$, and the periodic boundary conditions have been used.

Theorem 3.1. Let $\left(q_{h}^{n}, r_{h}^{n}, u_{h}^{n}\right)$ be obtained from the $D G$ formulation (48). Then both invariants $E_{1}$ and $E_{3}$ are preserved for the $D G$ scheme in the sense that

$$
D_{t} \int u_{h}^{n} d x=0, \quad D_{t} \int\left(\frac{1}{2}\left(r_{h}^{n}\right)^{2}-F\left(u_{h}^{n}\right)\right) d x=0 .
$$

Proof. To see the scheme preserves $E_{1}$ we simply take $\xi=1$ in (48a) to obtain $D_{t} \int u_{h}^{n} d x=0$.

To show the conservation of $E_{3}$, we take $\xi=A q_{h}^{n}$ in (48a), $\eta=-D_{t} u_{h}^{n}$ in (48b) and $\rho=A r_{h}^{n}$ in $D_{t}(48 \mathrm{c})$ to obtain

$$
\begin{aligned}
& \int D_{t} u_{h}^{n} A q^{n} d x=-\int A q_{h}^{n} q_{h x}^{n} d x-\sum_{j}\left(\widehat{A q_{h}^{n}}\left[q^{n}\right]\right)_{j+1 / 2}, \\
& -\int A q^{n} D_{t} u_{h}^{n} d x=-\int A r_{h}^{n} D_{t} u_{h x}^{n} d x-\sum_{j} \widehat{A r_{h}^{n}}\left[D_{t} u_{h}^{n}\right]_{j+1 / 2}+D_{t} \int F\left(u_{h}^{n}\right) d x, \\
& \int D_{t} r_{h}^{n} A r_{h}^{n} d x+\int D_{t} u_{h}^{n}\left(A r_{h}^{n}\right)_{x} d x+\sum_{j} \widehat{D_{t} u_{h}^{n}}\left[A r_{h}^{n}\right]_{j+1 / 2}=0 .
\end{aligned}
$$

Adding these together and integrating the complete spatial derivatives out, we obtain

$$
\frac{d}{d t} \int\left(\frac{\left(r_{h}^{n}\right)^{2}}{2}-F\left(u_{h}^{n}\right)\right) d x=-\sum_{j=1}^{N}\left(\widehat{A r_{h}^{n}}\left[D_{t} u_{h}^{n}\right]+\widehat{D_{t} u_{h}^{n}}\left[A r_{h}^{n}\right]-\left[D_{t} u_{h}^{n} A r_{h}^{n}\right]\right)_{j+1 / 2}=0
$$

We compute numerical solutions for the KdV equation using our scheme (48). To solve the implicit scheme (48) numerically we use the Newton iteration.

\section{Numerical RESUlts}

In this section we provide numerical examples to illustrate the accuracy and capability of the method. With the aid of successive mesh refinements we have verified that, in all cases, the results shown are numerically convergent.

Example 4.1. We consider the IVP of the KdV equation

$$
\left\{\begin{aligned}
u_{t}+u u_{x}+\epsilon u_{x x x}=0, & x \in(0,1), t>0, \\
u(x, 0)=u_{0}(x), & x \in(0,1),
\end{aligned}\right.
$$

with periodic boundary conditions. The exact solution is the so-called cnoidal-wave solution,

$$
u(x, t)=a c n^{2}\left(4 K(m)\left(x-v t-x_{0}\right)\right),
$$


where $\operatorname{cn}(z)=c n(z \mid m)$ is the Jacobi elliptic function with modulus $m=0.9$ and the parameters have the values $a=192 m \epsilon K^{2}(m), v=64 \epsilon(2 m-1) K^{2}(m)$, and $x_{0}=1 / 2$. The function $K=K(m)$ is the complete elliptic integral of the first kind.

In this example, we take $\epsilon=1 / 24^{2}$, and the computational domain is divided into $N$ cells. We test the $P^{k}, k=0,1,2,3$ polynomial approximations on uniform meshes. For the time discretization, we employ the second order implict scheme (48). We first test the accuracy and convergence rate of the method. Table 1 reports the numerical errors and the orders of accuracy for $P^{k}$ approximation at $t=1$. We observe that the scheme achieves the optimal order for $k$ even, and suboptimal for $k$ odd; see also Example 5.4 in Table 3. This suboptimal convergence for polynomials of odd degree also occurs in the energy $\left(E_{2}\right)$ conserving DG scheme for solving the $\mathrm{KdV}$ equation, see [2, 40].

TABLE 1. Errors for Example 4.1 when using $P^{k}$ polynomials on a uniform mesh of $N$ cells. Final time $T=1$.

\begin{tabular}{||c|c|c|c|c|c||}
\hline \hline \multirow{4}{*}{$k=0$} & $\Delta t$ & $1.0 \mathrm{e}-2$ & $2.5 \mathrm{e}-3$ & $6.25 \mathrm{e}-4$ & $1.5625 \mathrm{e}-4$ \\
\cline { 2 - 6 } & $N$ & 20 & 40 & 80 & 160 \\
\cline { 2 - 6 } & $\left\|u-u_{h}\right\|$ & $4.60 \mathrm{e}-1$ & $2.00 \mathrm{e}-2$ & $5.63 \mathrm{e}-2$ & $2.04 \mathrm{e}-2$ \\
\cline { 2 - 6 } & order & - & 1.20 & 1.83 & 1.47 \\
\hline \multirow{4}{*}{$k=1$} & $\Delta t$ & $1.0 \mathrm{e}-2$ & $2.5 \mathrm{e}-3$ & $6.25 \mathrm{e}-4$ & $1.5625 \mathrm{e}-4$ \\
\cline { 2 - 6 } & $N$ & 20 & 40 & 80 & 160 \\
\cline { 2 - 6 } & $\left\|u-u_{h}\right\|$ & $1.01 \mathrm{e}-1$ & $3.71 \mathrm{e}-2$ & $8.95 \mathrm{e}-3$ & $3.60 \mathrm{e}-3$ \\
\cline { 2 - 6 } & order & - & 1.44 & 2.05 & 1.31 \\
\hline \multirow{4}{*}{$k=2$} & $\Delta t$ & $1.0 \mathrm{e}-2$ & $2.5 \mathrm{e}-3$ & $6.25 \mathrm{e}-4$ & $1.5625 \mathrm{e}-4$ \\
\cline { 2 - 6 } & $N$ & 20 & 40 & 80 & 160 \\
\cline { 2 - 6 } & $\left\|u-u_{h}\right\|$ & $4.36 \mathrm{e}-3$ & $3.76 \mathrm{e}-4$ & $3.18 \mathrm{e}-5$ & $3.60 \mathrm{e}-6$ \\
\cline { 2 - 6 } & order & - & 3.54 & 3.56 & 3.14 \\
\cline { 2 - 6 } & $\Delta t$ & $6.25 \mathrm{e}-4$ & $1.5625 \mathrm{e}-4$ & $3.90625 \mathrm{e}-05$ & $9.765625 \mathrm{e}-06$ \\
\cline { 2 - 6 } & $N$ & 80 & 160 & 320 & 640 \\
\cline { 2 - 6 } & $\left\|u-u_{h}\right\|$ & $2.11 \mathrm{e}-5$ & $2.10 \mathrm{e}-6$ & $1.55 \mathrm{e}-07$ & $1.03 \mathrm{e}-08$ \\
\cline { 2 - 6 } & order & - & 3.33 & 3.76 & 3.91 \\
\hline \hline
\end{tabular}

We now verify the conservative properties of the DG method. Using the Cnoidal-wave test problem on uniform mesh of 80 cells with $k=2$ and $\Delta t=0.001$, we carry out the computation up to $t=1000$. Fig. 1 presents the exact solution and the numerical solutions at times $t=0,200,500$ and $t=1000$, respectively, showing that the DG method gives a very good approximation to the exact solution. We also present the time evolution of the $L^{2}$-error and the three conserved integrals $E_{1}, E_{2}, E_{3}$ up to time $t=1000$ in Fig. 2. We see that $E_{1}, E_{3}$ are well preserved, so is $E_{2}$; also the $L^{2}$ error of the DG method increases linearly with time. This may reflect the fact that all the solitons travel with well-preserved shape in the numerical solution and thus the principle part of the numerical error is the phase error.

We further compare the $E_{2}$ preserving scheme introduced in [40] with our $E_{3}$ preserving scheme in Fig. 3, in terms of the $L^{2}$-error, the time history of $E_{2}$ and $E_{3}$, as well as the numerical solutions at times $t=200,500,1000$, respectively. We clearly see that our $E_{3}$ preserving scheme induces smaller $L^{2}$ error and smaller phase errors. Moreover, it preserves both $E_{2}$ and $E_{3}$, while the $E_{2}$ preserving scheme only preserves $E_{2}$, with large oscillaltions observed in the time history of $E_{3}$. The corresponding comparison data are listed in Table 2. 


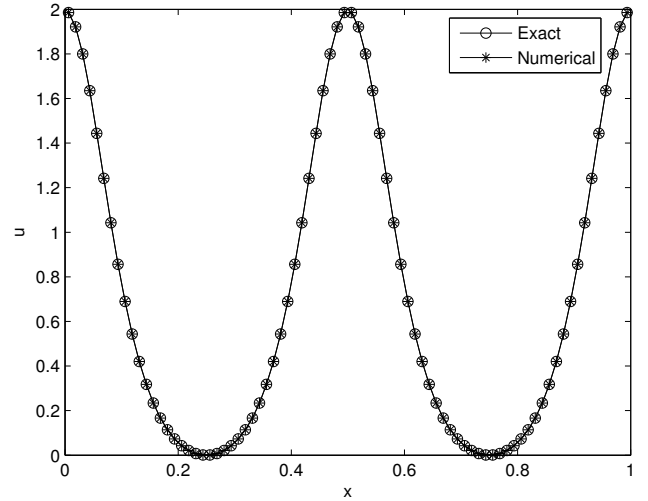

(a) $t=0$.

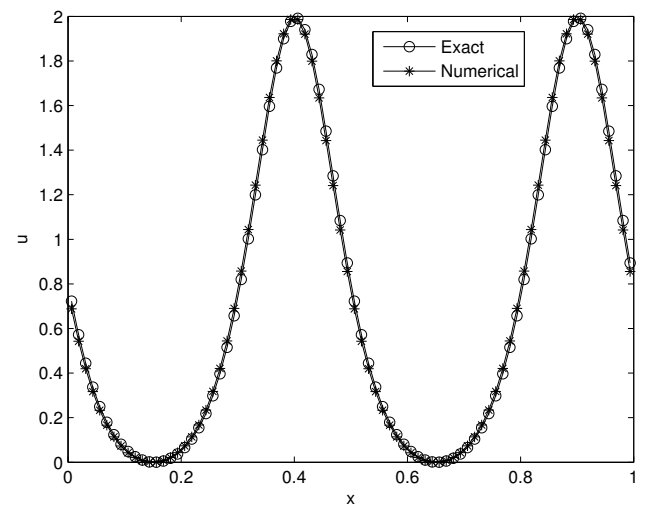

(c) $t=500$.

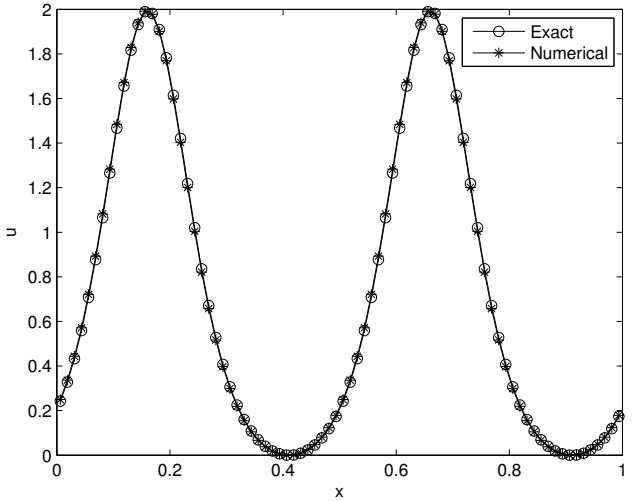

(b) $t=200$.

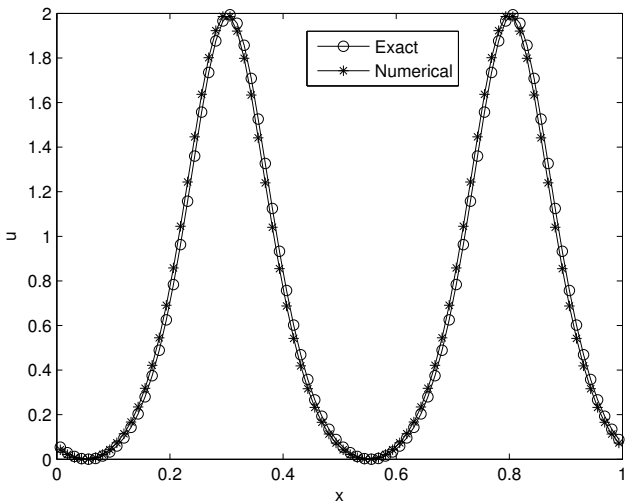

(d) $t=1000$.

Figure 1. Example 4.1, the solutions at $t=0,200,500$ and 1000 with $k=$ $2, \Delta t=1.0 e-03$ and 80 cells.

TABLE 2. The $E_{2}$ and $E_{3}$ of $E_{2}$ preserving and $E_{3}$ preserving schemes for Example 4.1 when using $P^{2}$ polynomials on a uniform mesh of 80 cell.

\begin{tabular}{||c|c|c|c|c|c||}
\hline \hline$E_{2}$ preserving scheme & $\mathrm{t}=0$ & $\mathrm{t}=200$ & $\mathrm{t}=500$ & $\mathrm{t}=900$ & $\mathrm{t}=1000$ \\
\hline$E_{2}(u)$ & 1.00729302749 & 1.00729302749 & 1.00729302749 & 1.00729302749 & 1.00729302749 \\
\hline$E_{2}\left(u_{h}\right)$ & 1.00729302690 & 1.00729302690 & 1.00729302690 & 1.00729302690 & 1.00729302690 \\
\hline$\frac{E_{2}\left(u_{h}\right)-E_{2}(u) \mid}{E_{2}(u)}$ & $5.8573 \mathrm{e}-10$ & $5.8573 \mathrm{e}-10$ & $5.8573 \mathrm{e}-10$ & $5.8573 \mathrm{e}-10$ & $5.8573 \mathrm{e}-10$ \\
\hline \hline$E_{3}$ preserving scheme & $\mathrm{t}=0$ & $\mathrm{t}=200$ & $\mathrm{t}=500$ & $\mathrm{t}=900$ & $\mathrm{t}=1000$ \\
\hline$E_{2}\left(u_{h}\right)$ & 1.00729302691 & 1.00729302692 & 1.00729302691 & 1.00729302692 & 1.00729302691 \\
\hline$\frac{E_{2}\left(u_{h}\right)-E_{2}(u) \mid}{E_{2}(u)}$ & $5.7580 \mathrm{e}-10$ & $5.6587 \mathrm{e}-10$ & $5.7580 \mathrm{e}-10$ & $5.6587 \mathrm{e}-10$ & $5.7580 \mathrm{e}-10$ \\
\hline \hline$E_{2}$ preserving scheme & $\mathrm{t}=0$ & $\mathrm{t}=200$ & $\mathrm{t}=500$ & $\mathrm{t}=900$ & $\mathrm{t}=1000$ \\
\hline$E_{3}\left(u_{h}\right)$ & -0.18912751410 & -0.19031815943 & -0.19004235096 & -0.18995786446 & -0.19051454372 \\
\hline \hline$E_{3}$ preserving scheme & $\mathrm{t}=0$ & $\mathrm{t}=200$ & $\mathrm{t}=500$ & $\mathrm{t}=900$ & $\mathrm{t}=1000$ \\
\hline$E_{3}\left(u_{h}\right)$ & -0.18925223716 & -0.18925223716 & -0.18925223716 & -0.18925223716 & -0.18925223716 \\
\hline \hline
\end{tabular}

Example 4.2. Consider again the $K d V$ equation (49) with $\epsilon=1$, and $u(x, 0)$ as the initial data, the exact solution is of the two soliton waves

$$
u(x, t)=12 \frac{k_{1}^{2} e^{\theta_{1}}+k_{2}^{2} e^{\theta_{2}}+2\left(k_{2}-k_{1}\right)^{2} e^{\theta_{1}+\theta_{2}}+a^{2}\left(k_{2}^{2} e^{\theta_{1}}+k_{1}^{2} e^{\theta_{2}}\right) e^{\theta_{1}+\theta_{2}}}{\left(1+e^{\theta_{1}}+e^{\theta_{2}}+a^{2} e^{\theta_{1}+\theta_{2}}\right)^{2}},
$$




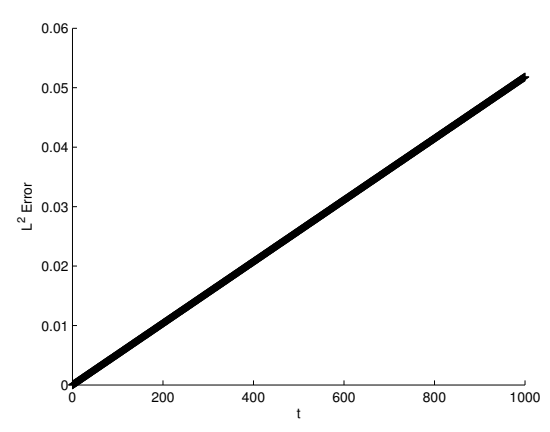

(a) $L^{2}$ errors.

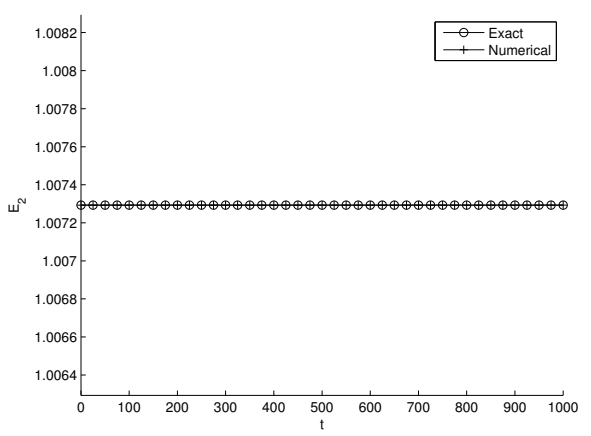

(c) $E_{2}$ history.

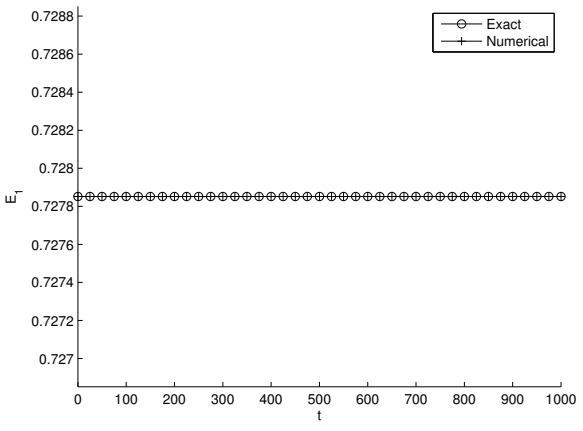

(b) $E_{1}$ history.

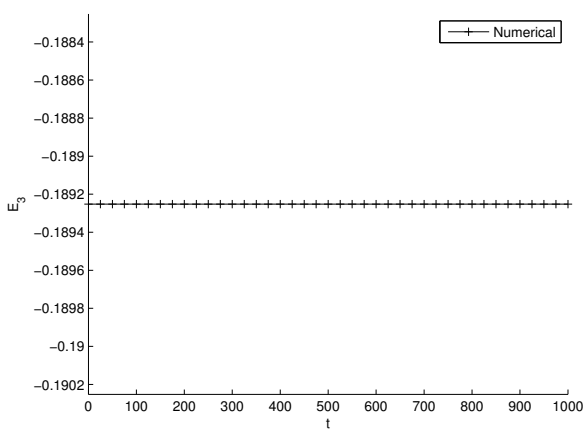

(d) $E_{3}$ history.

Figure 2. Example 4.1, time history of the $L^{2}$ error, $E_{1}, E_{2}$ and $E_{3}$ plots using the DG methods with $k=2, \Delta t=1.0 e-03$ and 80 cells.

where

$$
\begin{aligned}
& k_{1}=0.4, \quad k_{2}=0.6, \quad a^{2}=\left(\frac{k_{1}-k_{2}}{k_{1}+k_{2}}\right)^{2}=\frac{1}{25}, \\
& \theta_{1}=k_{1} x-k_{1}^{3} t+x_{1}, \quad \theta_{2}=k_{2} x-k_{2}^{3} t+x_{2}, \\
& x_{1}=4, \quad x_{2}=15 .
\end{aligned}
$$

This example is derived from [24]. The solution region is taken to be $[-40,40]$ with periodic boundary conditions. Initially we have two solitons, the left the tall one and the right short one. Both solitons travel from left to right, and speed of the tall one is bigger than the speed of the short one. We perform computation for $k=2$ and $\Delta t=0.1$. The grid is taken to be of 400 cells and the computation is implemented up to $t=120$. The plots of the exact solution and numerical solution at the times are displayed in Fig. 4. It shows that the numerical solution agrees quite well with the exact one.

Example 4.3. Consider the Zabusky-Kruskal's problem, setting the dispersion coefficient $\epsilon=$ $0.022^{2}$ and

$$
u(x, 0)=\cos (\pi x),
$$

with periodic boundary conditions. The solution starts with a cosine wave and later on develops a train of 8 solitons which travel at different speeds and interact with each other, see [41] for detailed description of the solution. There are several critical moments in the development of the solution: (1) $t=t_{B}=\frac{1}{\pi}$ when the solution is about to breakdown, (2) $t=3.6 t_{B}$ when a train of 8 solitons have been developed. 


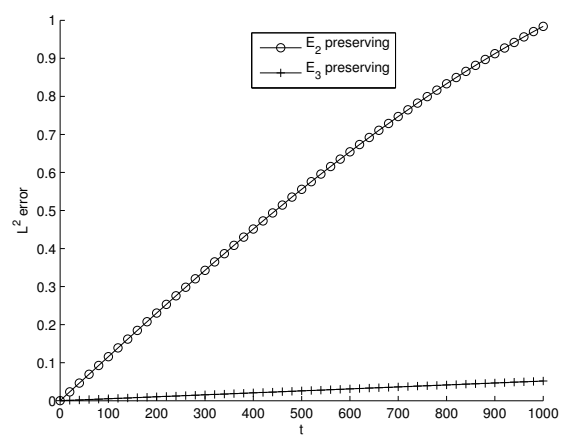

(a) $L^{2}$ errors.

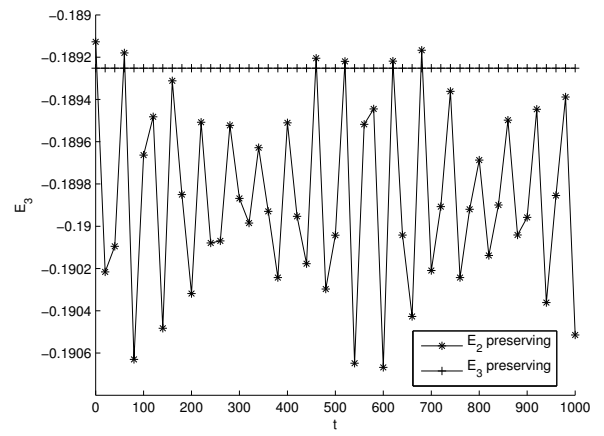

(c) $E_{3}$ history.

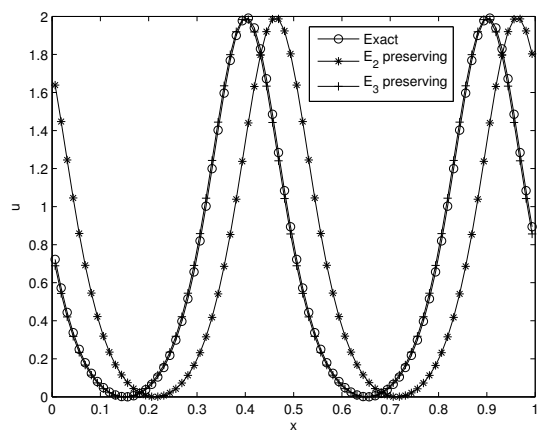

(e) Solution at $t=500$.

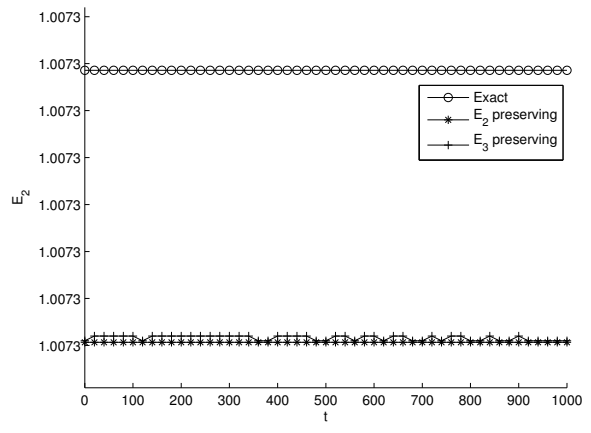

(b) $E_{2}$ history.

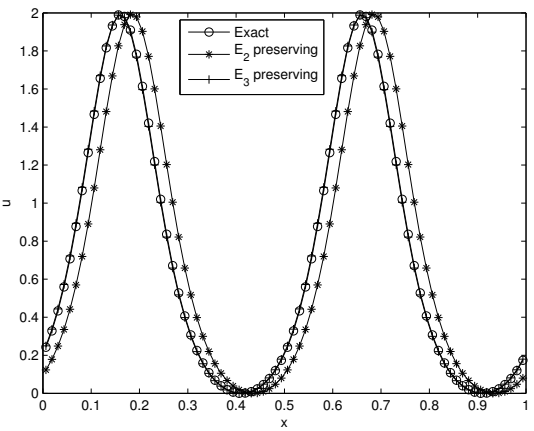

(d) Solution at $t=200$.

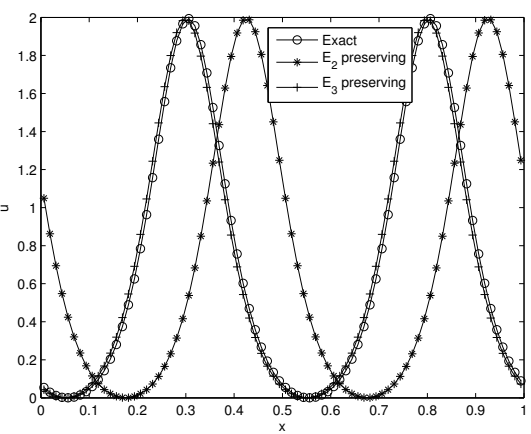

(f) Solution at $t=1000$.

FiguRE 3. Example 4.1, the performance of the $E_{2}$ preserving and $E_{3}$ preserving schemes comparisons with $k=2, \Delta t=1.0 e-03$ and 80 cells.

We perform computation on a uniform mesh of 400 cells with $k=2$ and $\Delta t=\frac{0.005}{\pi}$. The initial values and the numerical solution at the two times $t=t_{B}, 3.6 t_{B}$ are plotted in Figure 5. It shows that all of them agree quite well with the numerical result obtained by Zabusky and Kruskal in 1965, see [41], as well as the other numerical schemes in [2, 24, 40].

Now, we come to show that our method can satisfy the first three conservation laws for the linear $\mathrm{KdV}$ equation. 


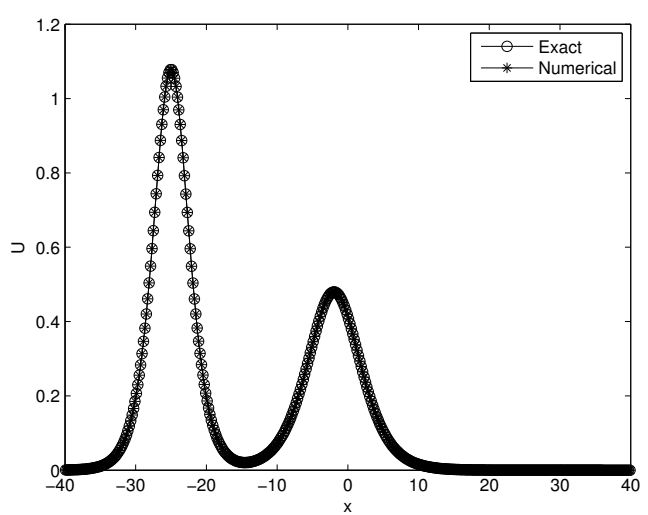

(a) $t=0$.

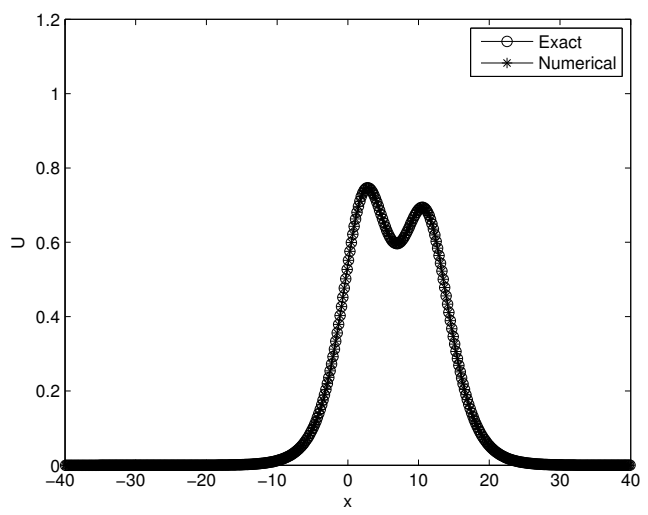

(c) $t=80$.

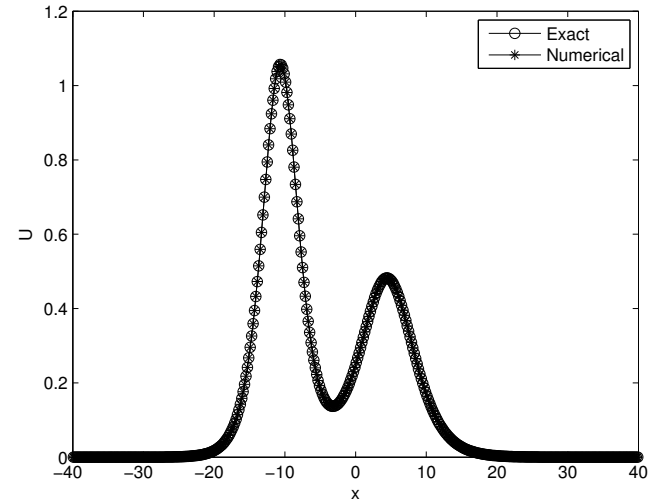

(b) $t=40$.

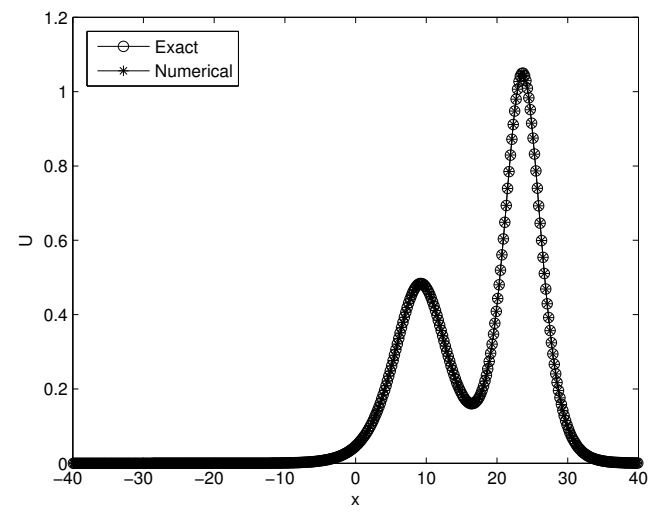

(d) $t=120$.

Figure 4. Example 4.2, the solutions at $t=0,40,80$ and 120 with $k=2, \Delta t=$ 0.1 and 400 cells.

Example 4.4. We solve the linear $K d V$ equation given by

$$
\left\{\begin{array}{l}
u_{t}-u_{x}+u_{x x x}=0, \\
u(x, 0)=\sin (x), \\
u(0, t)=u(2 \pi, t) .
\end{array}\right.
$$

The exact solution is

$$
u(x, t)=\sin (x+2 t) .
$$

Firstly, we use this solution to check the accuracy and convergence rate of the method. We perform the computation on the grids of $10,20,40,80,160$ cells, respectively, up to $t=1$. The $L^{2}$ errors and the corresponding convergence rates are presented in Table 3. We can clearly see from the table that our method achieves optimal order for polynomials of even degree, i.e., $k=0,2$ in Table 3 .

Secondly, we use this solution to test the preserving feature of our method in long-time simulation. We perform the computation with $(k, N, \Delta t)=(1,80,6.25 e-04)$ and $(k, N, \Delta t)=$ $(2,40,2.5 e-03)$, respectively. The numerical results are displayed in Fig. 6-Fig. 9. We can see from Fig. 6 and Fig. 8 that our method is quite stable in long-time simulation. The phase error in Fig. 6 with $k=1$ tends bigger as time becomes large, yet the phase error at the same 


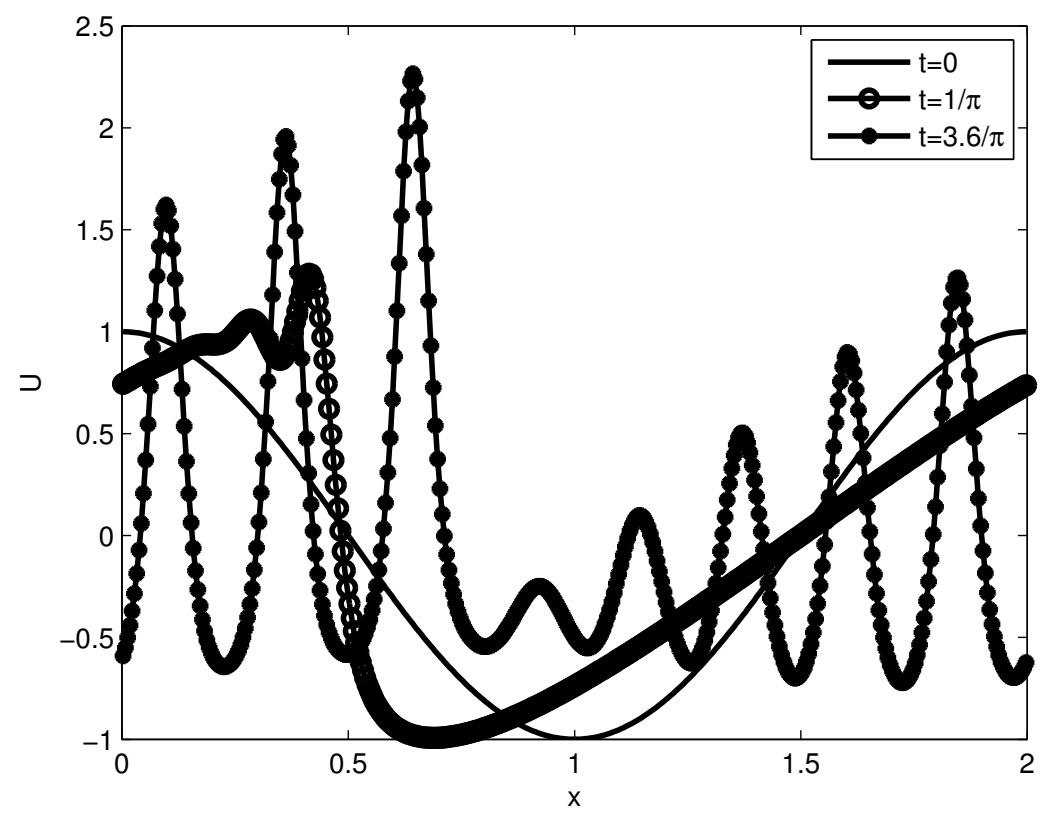

Figure 5. Example 4.3 , the solutions at $t=0,1 / \pi$ and $3.6 / \pi$ with $k=2, \Delta t=$ $0.005 / \pi$ and 400 cells.

TABLE 3. Errors for Example 4.4 when using $P^{k}$ polynomials on a uniform mesh of $N$ cells. Final time $T=1$.

\begin{tabular}{||c|c|c|c|c|c|c||}
\hline \hline \multirow{4}{*}{$k=0$} & $\Delta t$ & $4.0 \mathrm{e}-2$ & $1.0 \mathrm{e}-2$ & $2.5 \mathrm{e}-3$ & $6.25 \mathrm{e}-4$ & $1.5625 \mathrm{e}-4$ \\
\cline { 2 - 7 } & $N$ & 10 & 20 & 40 & 80 & 160 \\
\cline { 2 - 7 } & $\left\|u-u_{h}\right\|$ & $4.69 \mathrm{e}-1$ & $1.97 \mathrm{e}-1$ & $8.54 \mathrm{e}-2$ & $4.08 \mathrm{e}-2$ & $2.02 \mathrm{e}-2$ \\
\cline { 2 - 7 } & order & - & 1.25 & 1.21 & 1.06 & 1.02 \\
\hline \multirow{4}{*}{$k=1$} & $\Delta t$ & $4.0 \mathrm{e}-2$ & $1.0 \mathrm{e}-2$ & $2.5 \mathrm{e}-3$ & $6.25 \mathrm{e}-4$ & $1.5625 \mathrm{e}-4$ \\
\cline { 2 - 7 } & $N$ & 10 & 20 & 40 & 80 & 160 \\
\cline { 2 - 7 } & $\left\|u-u_{h}\right\|$ & $1.18 \mathrm{e}-1$ & $2.44 \mathrm{e}-2$ & $8.13 \mathrm{e}-3$ & $5.35 \mathrm{e}-3$ & $2.84 \mathrm{e}-3$ \\
\cline { 2 - 7 } & order & - & 2.23 & 1.59 & 0.61 & 0.91 \\
\hline \multirow{4}{*}{$k=3$} & $\Delta t$ & $4.0 \mathrm{e}-2$ & $1.0 \mathrm{e}-2$ & $2.5 \mathrm{e}-3$ & $6.25 \mathrm{e}-4$ & $1.5625 \mathrm{e}-4$ \\
\cline { 2 - 7 } & $N$ & 10 & 20 & 40 & 80 & 160 \\
\cline { 2 - 7 } & $\left\|u-u_{h}\right\|$ & $2.41 \mathrm{e}-3$ & $2.13 \mathrm{e}-4$ & $2.30 \mathrm{e}-5$ & $2.7 \mathrm{e}-6$ & $3.0 \mathrm{e}-7$ \\
\cline { 2 - 7 } & order & - & 3.50 & 3.21 & 3.09 & 3.17 \\
\cline { 2 - 7 } & $\Delta t$ & $4.0 \mathrm{e}-2$ & $1.0 \mathrm{e}-2$ & $2.5 \mathrm{e}-3$ & $6.25 \mathrm{e}-4$ & $1.5625 \mathrm{e}-4$ \\
\hline & $N$ & 10 & 20 & 40 & 80 & 160 \\
\cline { 2 - 7 } & $\left\|u-u_{h}\right\|$ & $1.90 \mathrm{e}-3$ & $1.24 \mathrm{e}-4$ & $7.55 \mathrm{e}-6$ & $8.15 \mathrm{e}-7$ & $3.28 \mathrm{e}-08$ \\
\cline { 2 - 7 } & order & - & 3.93 & 4.01 & 3.21 & 4.64 \\
\hline \hline
\end{tabular}

simulation time is almost invisible when using $k=2$, see Fig. 8. Fig. 7 and Fig. 9 show that the $L^{2}$ error increases linearly with time, and the three qualities $E_{1}, E_{2}, E_{3}$ are all preserved. 


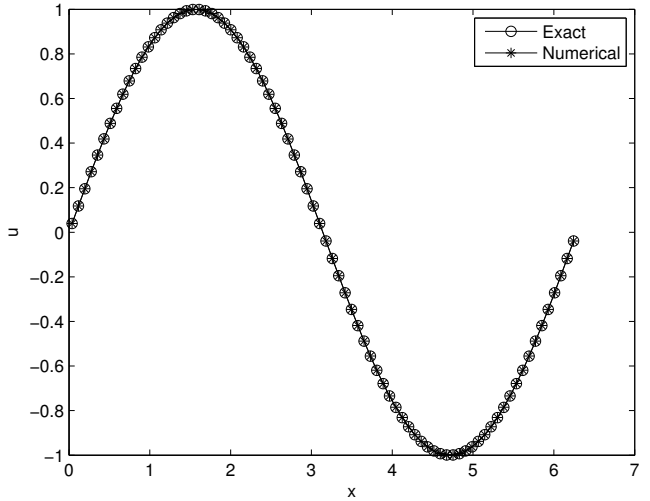

(a) $t=0$.

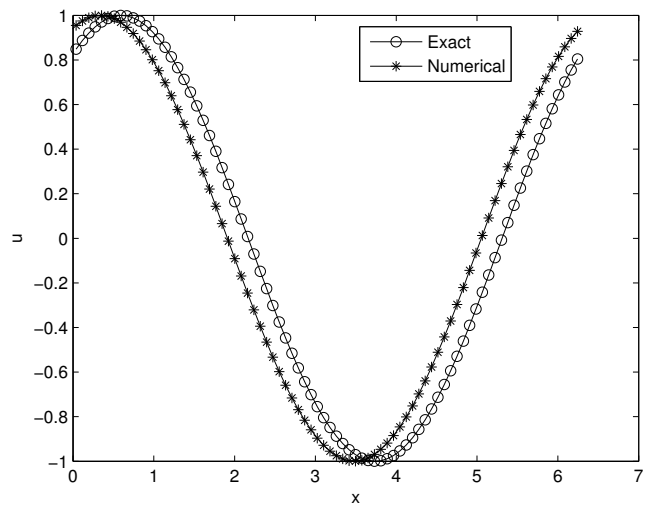

(c) $t=500$.

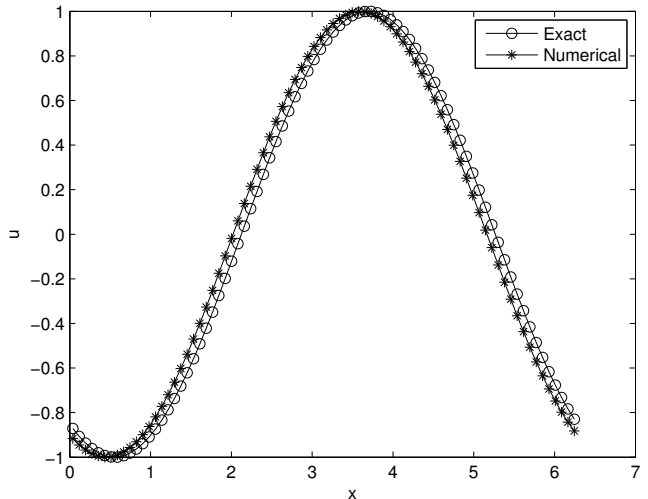

(b) $t=200$.

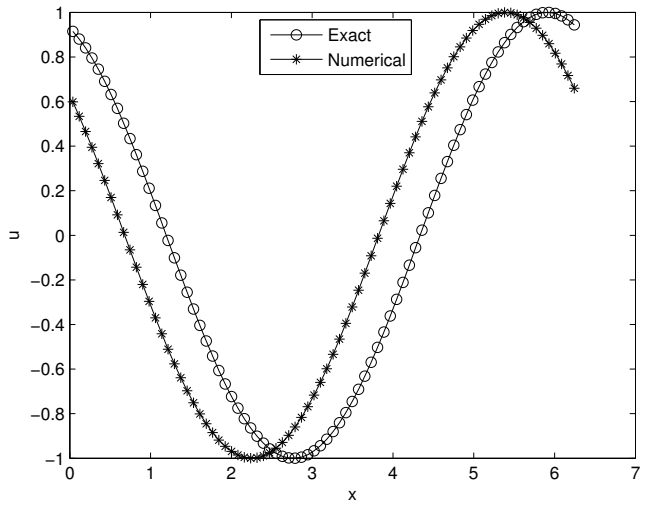

(d) $t=1000$.

Figure 6. Example 4.4, the solutions at $t=0,200,500$ and 1000 with $k=$ $1, \Delta t=6.25 e-04$ and 80 cells.

\section{Concluding Remarks}

In this paper, we developed and analyzed a Hamiltonian preserving DG method for solving the generalized KdV equation. Both semi-discrete and fully discrete DG schemes are shown to preserve $E_{1}$ and $E_{3}$. The better performance of the $E_{3}$-preserving scheme than the $E_{2}$-preserving scheme is shown through numerical comparison.

For wave equations, usually it is difficult to obtain DG schemes which can preserve more conservative integrals while still maintaining optimal high order of accuracy. Our scheme when applied to the linearized $\mathrm{KdV}$ equation preserves all the first three conserved integrals and has the proven optimal error estimates for polynomial elements of even degree. Our numerical experiments have demonstrated both high accuracy of convergence and preservation of all three conserved integrals for the generalized $\mathrm{KdV}$ equation. It is confirmed by numerical simulations that the Hamiltonian preserving property is important for minimizing phase and amplitude errors after long time simulation 


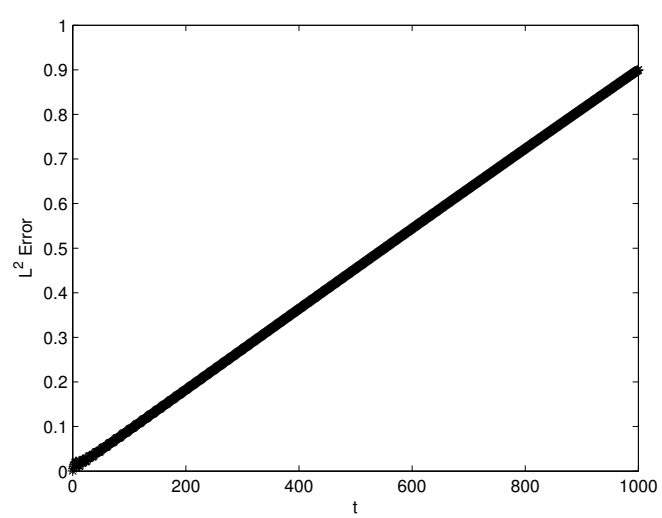

(a) $L^{2}$ errors.

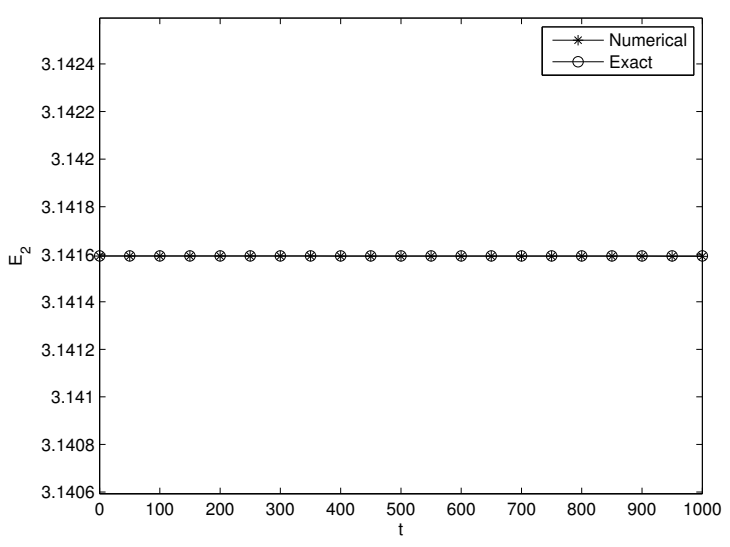

(c) $E_{2}$ history.

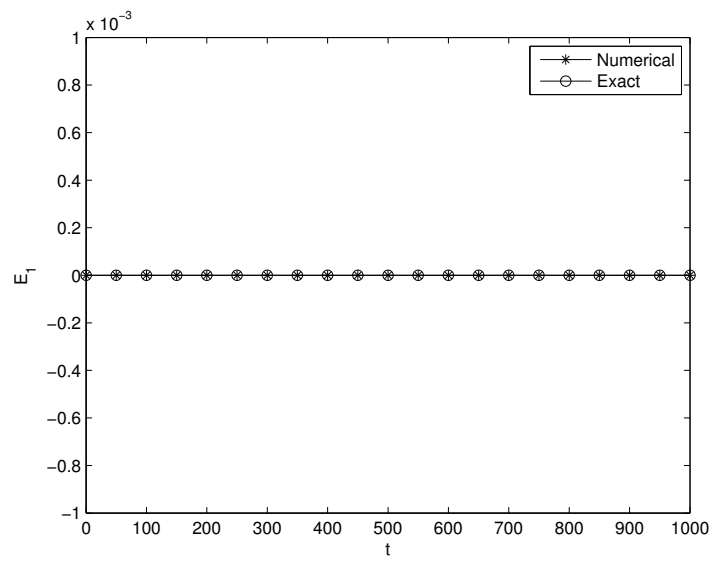

(b) $E_{1}$ history.

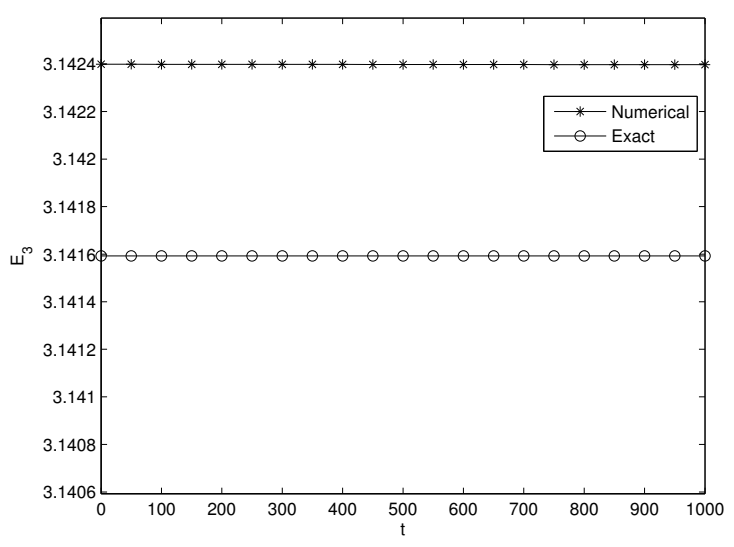

(d) $E_{3}$ history.

FiguRE 7. Example 4.4, time history of the $L^{2}$ error, $E_{1}, E_{2}$ and $E_{3}$ plots using the DG methods with $k=1, \Delta t=6.25 e-04$ and 80 cells.

\section{ACKNOWLEDGMENTS}

This research was partially supported by the National Science Foundation under grant DMS1312636 and the KI-Net research network. Yi's research was supported by NSFC Project (11201397, 91430213) and Hunan Provincial NSF Project (2015JJ2145).

\section{REFERENCES}

[1] D.N. Arnold and R. Winther. A superconvergent finite element method for the Korteweg-de Vries equation. Math. Comp., 38:23-36, 1982.

[2] J. Bona, H. Chen, O. Karakashian, and Y. Xing. Conservative, discontinuous-Galerkin methods for the generalized Korteweg-de Vries equation. Math. Comp., 82(283):1401-1432, 2013.

[3] Y. Cheng and C.W. Shu. A discontinuous Galerkin finite element method for time dependent partial differential equations with higher order derivatives. Math. Comp., 77:699-730, 2008.

[4] B. Cockburn and C.W. Shu. TVB Runge-Kutta local projection discontinuous Galerkin finite element method for conservation laws II: general framework. Math. Comp., 52:411-435, 1989.

[5] B. Cockburn and S.Y. Lin and C.W. Shu. TVB Runge-Kutta local projection discontinuous Galerkin finite element method for conservation laws III: one dimensional systems. J. Comput. Phys., 84:90-113, 1989. 


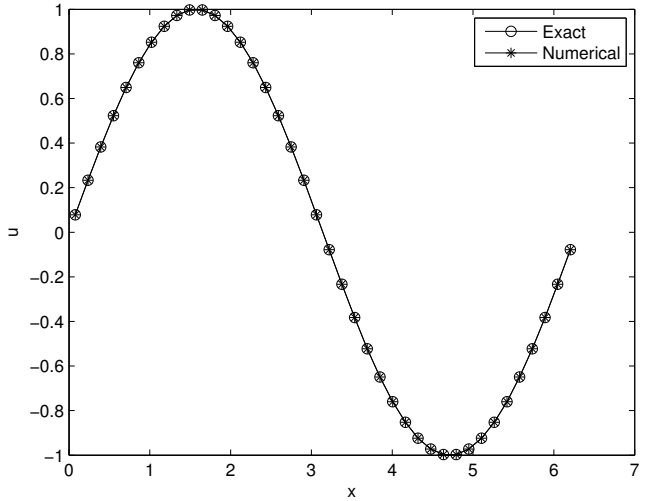

(a) $t=0$.

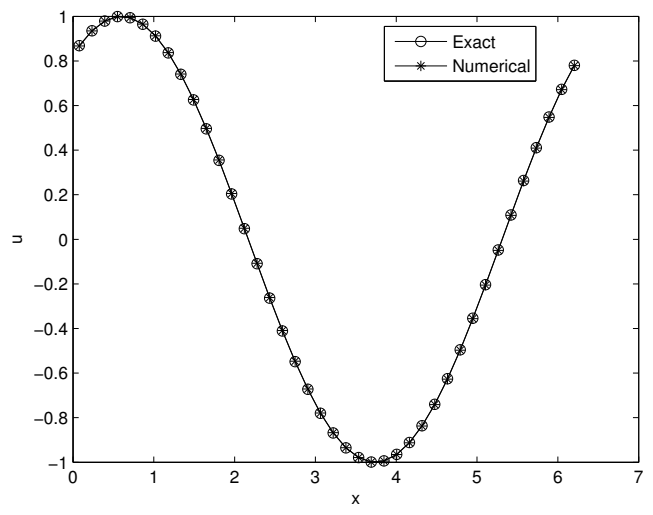

(c) $t=500$.

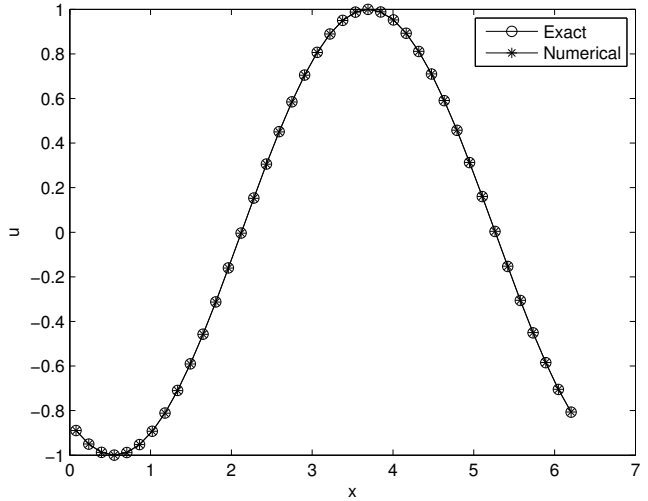

(b) $t=200$.

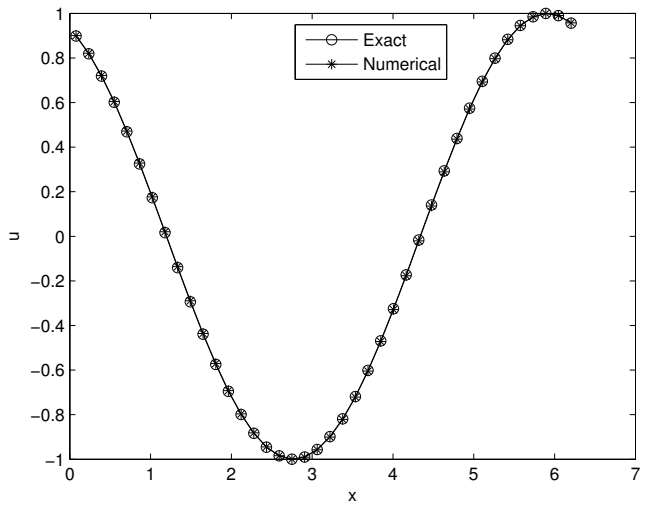

(d) $t=1000$.

Figure 8. Example 4.4, the solutions at $t=0,200,500$ and 1000 with $k=$ $2, \Delta t=2.5 e-03$ and 40 cells.

[6] B. Cockburn and S. Hou and C.W. Shu. The Runge-Kutta local projection discontinuous Galerkin finite element method for conservation laws IV: the multidimensional case. Math. Comput., 54:545-581, 1990.

[7] B. Cockburn and C.W. Shu. The Runge-Kutta discontinuous Galerkin method for conservation laws V: multidimensional systems. J. Comput. Phys., 141:199-224, 1998.

[8] L. Faddeev and V. E. Zakharov. Korteweg-de Vries equation as completely integrable Hamiltonian system. Funk. Anal. Priloz., 5: 18-27, 1971. (In Russian.)

[9] B. Fornberg and GB Whitham. A numerical and theoretical study of certain nonlinear wave phenomena. Philosophical Transactions of the Royal Society of London. Series A, Mathematical and Physical Sciences, 289:373-404, 1978.

[10] D. Furihata. Finite difference schemes for $\frac{\partial u}{\partial t}=\left(\frac{\partial}{\partial x}\right)^{\alpha} \frac{\delta g}{\delta u}$ that inherit energy conservation or dissipation property. J. Comput. Phys., 156:181-205, 1999.

[11] C. S. Gardner. Korteweg-de Vries equation and generalizations. IV: The Korteweg-de Vries equation as a Hamiltonian system. J. Mathematical Phys., 12: 1548-1551, 1971.

[12] K. Goda. Numerical studies on recurrence of the Korteweg-de Vries equation. J. Phys. Soc. Japan, 42:10401046, 1977.

[13] I.S. Greig and J.L. Morris. A hopscotch method for the Korteweg-de Vries equation. J. Comput. Phys., 20:64-80, 1976.

[14] B. Guo and J. Shen. On spectral approximations using modified Legendre rational functions: application to the Korteweg-de Vries equation on the half line. Indiana University Mathematics Journal, 50:181-204, 2001.

[15] Helge Holden, Kenneth H. Karlsen, Nils Henrik Risebro, and Terence Tao. Operator splitting for the KdV equation. Math. Comp., 80(274):821-846, 2011. 


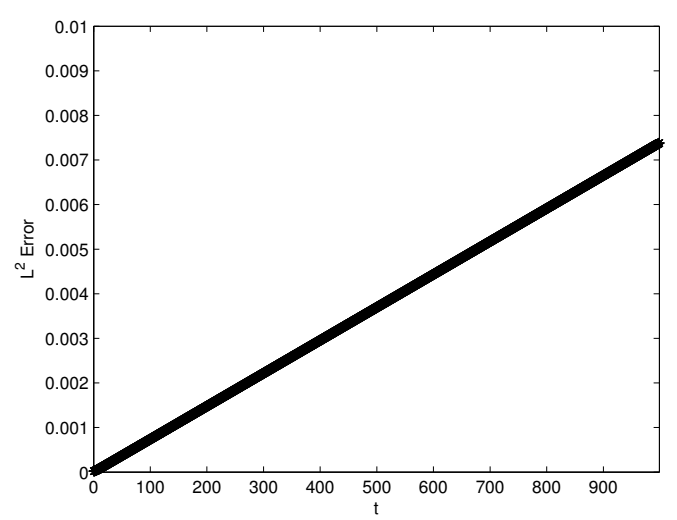

(a) $L^{2}$ errors.

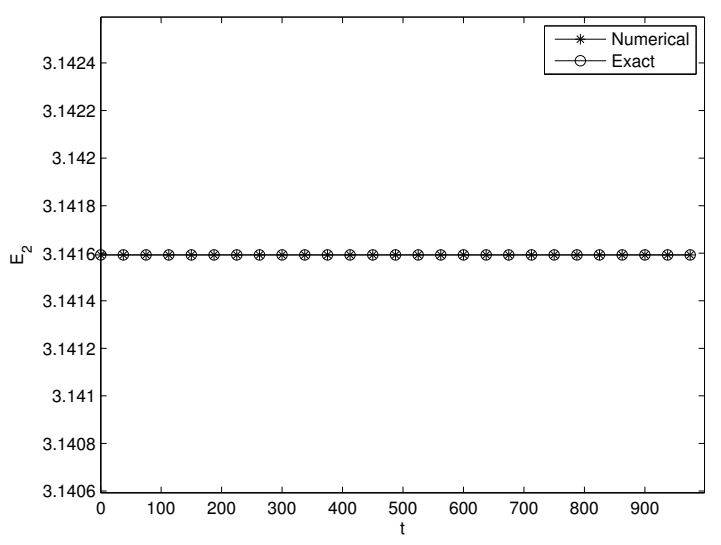

(c) $E_{2}$ history.

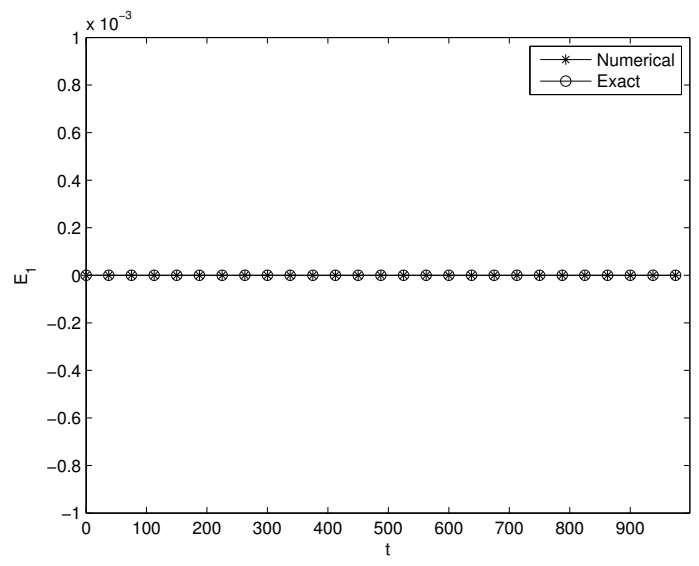

(b) $E_{1}$ history.

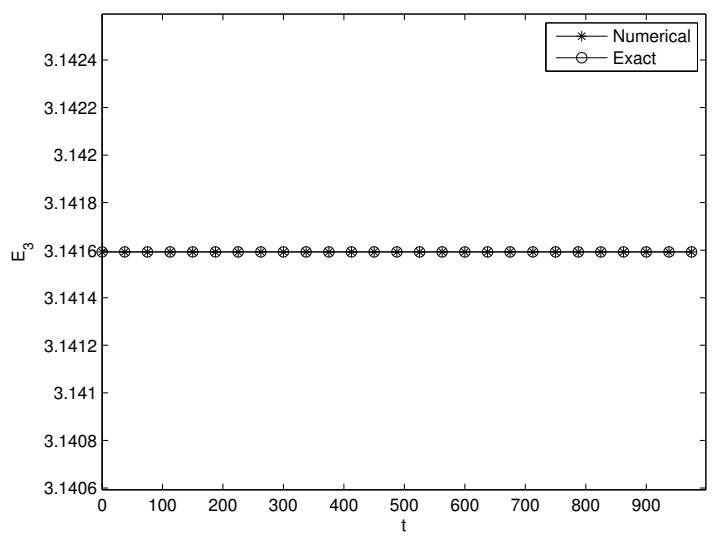

(d) $E_{3}$ history.

Figure 9. Example 4.4, time history of the $L^{2}$ error, $E_{1}, E_{2}$ and $E_{3}$ plots using the DG methods with $k=2, \Delta t=2.5 e-03$ and 40 cells.

[16] H. Holden, K.H. Karlsen, and N.H. Risebro. Operator splitting methods for generalized Korteweg-de Vries equations. J. Comput. Phys., 153:203-222, 1999.

[17] L. Ji and Y. Xu, Optimal error estimates of the local discontinuous Galerkin method for Willmore flow of graphs on Cartesian meshes, Int. J. Numer. Anal. Model., 8:252-283, 2011.

[18] D.J. Korteweg and G. de Vries. On the change of form of long waves advancing in a rectangular canal, and on a new type of long stationary waves. Philosophical Magazine, 39:422-443, 1895.

[19] J. Li and M.R. Visbal. High-order compact schemes for nonlinear dispersive waves. J. Sci. Comput., 26:1-23, 2006.

[20] P. Lax. Integrals of nonlinear equations of evolution and solitary waves. Comm. Pure Applied Math., 21:467490, 1968.

[21] H. Liu, Y.Q. Huang and N.Y. Yi. A direct discontinuous Galerkin method for the Degasperis-Procesi equation. Methods and Applications of Analysis, 21:83-106, 2014.

[22] H. Liu and J. Yan. A local discontinuous Galerkin method for the Korteweg-de Vries equation with boundary effect. J. Comput. Phys., 215:197-218, 2006.

[23] W. Huang and D.M. Sloan. The pseudospectral method for third-order differential equations. SIAM J. Numer. Anal., 29:1626-1647, 1992.

[24] Y.F. Cui and D.K. Mao. Numerical method satisfying the first two conservation laws for the Korteweg-de Vries equation. J. Comput. Phys., 227:376-399, 2007. 
[25] H. Ma and W. Sun. A Legendre-Petrov-Galerkin and Chebyshev collocation method for third-order differential equations. SIAM J. Numer. Anal., 38:1425-1438, 2001.

[26] H. Ma and W. Sun. Optimal error estimates of the Legendre-Petrov-Galerkin method for the Korteweg-de Vries equation. SIAM J. Numer. Anal., 39:1380-1394, 2002.

[27] R.M. Miura, C.S. Gardner and M.D. Kruskal. Korteweg-de Vries equation and generalizations ii: existence of conservation laws and constants of motion. J. Comput. Phys., 9: 1204-1209, 1968.

[28] W. Reed and T. Hill. Triangular mesh methods for the neutrontransport equation, La-ur-73-479, Los Alamos Scientific Laboratory, 1973.

[29] J.M. Sanz-Serna and I. Christie. Petrov-Galerkin methods for nonlinear dispersive wave. J. Comput. Phys., 39:94-102, 1981.

[30] J. Shen. A new dual-Petrov-Galerkin method for third and higher odd-order differential equations: application to the KdV equation. SIAM J. Numer. Anal., 41:1595-1619, 2004.

[31] R. Winther. A conservative finite element method for the Korteweg-de Vries equation. Math. Comp., 34:2343, 1980.

[32] X.L. Xie. Large time-stepping methods for higher order time-dependent evolution equations. PhD thesis, Iowa State University, Ames, IA, 2008.

[33] Y. Xu and C.W. Shu. Local discontinuous Galerkin methods for the Degasperis-Procesi equation. Comm. Comput. Phys., 10(2):474-508, 2011.

[34] Y. Xu and C.W. Shu. Local discontinuous Galerkin methods for two classes of two-dimensional nonlinear wave equations. Physica D: Nonlinear phenomena, 208:21-58, 2005.

[35] Y. Xu and C.W. Shu. Error estimates of the semi-discrete local discontinuous Galerkin method for nonlinear convection-diffusion and $\mathrm{KdV}$ equations. Computer Methods in Applied Mechanics and Engineering, 196:3805-3822, 2007.

[36] Y. Xu and C.W. Shu. A local discontinuous Galerkin method for the Camassa-Holm equation. SIAM J. Numer. Anal., 46: 1998-2021, 2008.

[37] Y. Xu and C.W. Shu. Local discontinuous Galerkin method for higher order time-dependent partial differential equations. Commun. Comput. Phys., 7: 1-46, 2010.

[38] Y. Xu and C.-W. Shu, Optimal error estimates of the semi-discrete local discontinuous Galerkin methods for high order wave equations. SIAM J. Numer. Anal., 50(1):79-104, 2012.

[39] J. Yan and C.W. Shu. A local discontinuous Galerkin method for KdV type equations. SIAM J. Numer. Anal., 40:769-791, 2002.

[40] N. Yi, Y. Huang and H. Liu. A direct discontinuous Galerkin method for the generalized Korteweg-de Vries equation: Energy conservation and boundary effect. J. Comput. Phys. 242:351-366, 2013.

[41] N.J. Zabusky and M.D. Kruskal. Interactions of "solitons" in a collisionless plasma and the recurrence of initial states, Phys. Rev. Lett., 15:240-243, 1965.

${ }^{\dagger}$ Iowa State University, Mathematics Department, Ames, IA 50011

E-mail address: hliu@iastate.edu

${ }^{\dagger}$ School of Mathematics and Computational Science, Xiangtan University, Xiangtan 411105, P.R.CHINA

E-mail address: yinianyu365109@126.com 